\title{
Los mármoles cálcicos de El Escorial (complejo metamórfico Cushamen, macizo norpatagónico): caracterización isotópica de ${ }^{87} \mathrm{Sr} /{ }^{86} \mathrm{Sr}$ y edad de sedimentación
}

\author{
*Juan A. Murra ${ }^{1}$, Edgardo G. Baldo ${ }^{1}$, Sebastián O. Verdecchia ${ }^{1}$, \\ Carlos D. Ramacciotti ${ }^{1},{ }^{\dagger}$ Carmen Galindo ${ }^{2}$
}
Centro de Investigaciones en Ciencias de la Tierra (CICTERRA), Universidad Nacional de Córdoba (CONICET-UNC), Avda. Vélez Sársfield 1611 (5000), Córdoba, Argentina. juan.murra@unc.edu.ar; edgardo.baldo@unc.edu.ar; sverdecchia@unc.edu.ar; carlos.ramacciotti@unc.edu.ar
2 Departamento de Petrología y Geoquímica, Univerdidad Complutense de Madrid, Instituto de Geociencias (UCM, CSIC), 28040 Madrid, España.

*Autor para correspondencia: juan.murra@unc.edu.ar

\begin{abstract}
RESUMEN. Los mármoles de El Escorial (Complejo Metamórfico Cushamen) se encuentran asociados a anfibolitas que forman septos metamórficos dentro de los granitoides pérmicos de la Formación Mamil Choique (261-286 Ma). El metamorfismo de los septos, determinado en gneises migmáticos en facies de granulita aflorantes $120 \mathrm{~km}$ al suroeste de El Escorial, ocurre a los $311 \pm 27 \mathrm{Ma}$ (método CHIME en monacita). Los mármoles son de composición calcítica (calcita $>95 \%$, R.I.: 0,5 a $2,5 \%)$ y presentan razones $\mathrm{de}^{87} \mathrm{Sr} /{ }^{86} \mathrm{Sr}$ entre 0,70768 y $0,70825(\mathrm{n}=10)$. Los datos aportados en este trabajo, sumado a contribuciones previas, permiten acotar la edad de sedimentación de las sucesiones silícico-carbonáticas del Complejo Metamórfico Cushamen entre los ca. 385 y 335 Ma. Esto sugiere la existencia de una plataforma mixta (carbonática-siliciclástica) al menos en la porción más austral del suroeste de Gondwana entre el Devónico Medio y el Carbonífero inferior (Misisipiano Medio).
\end{abstract}

Palabras clave: Mármol, Isótopos de Sr, Macizo Norpatagónico, Suroeste de Gondwana.

\begin{abstract}
El Escorial calcitic marbles (Cushamen Metamorphic Complex, Macizo Norpatagónico): ${ }^{87} \mathrm{Sr} /{ }^{86} \mathrm{Sr}$ isotopic characterization and age of sedimentation. The El Escorial marbles (Cushamen Metamorphic Complex) along with amphibolites form metamorphic septa within the permian granitoids of the Mamil Choique Formation (261-286 Ma). The metamorphism, determined in granulite facies migmatic gneisses septa cropping out $120 \mathrm{~km}$ southwest of El Escorial, occurs at $311 \pm 27 \mathrm{Ma}$ (CHIME method in monazite). The marbles are calcitic (calcite $>95 \%$, R.I.: 0.5 to $2.5 \%)$ and show ${ }^{87} \mathrm{Sr} /{ }^{86} \mathrm{Sr}$ ratios between 0.70768 and $0.70825(\mathrm{n}=10)$. The data provided in this work, added to previous contributions, allow to constraints the sedimentation age of the silicic-carbonate successions of the Cushamen Metamorphic Complex between $c a .385$ and $335 \mathrm{Ma}$. This suggests the existence of a mixed carbonatesiliciclastic platform at least in the southernmost portion of southwestern Gondwana between Middle Devonian and early Carboniferous (Middle Mississippian).
\end{abstract}




\section{Introducción}

Las curvas de variaciones seculares de las composiciones isotópicas de $\mathrm{Sr}, \mathrm{C}$ y $\mathrm{O}$ para el agua oceánica en el mundo han sido establecidas con suficiente precisión, lo que hace factible la correlación temporal de carbonatos y rocas metacarbonatadas marinas, en los cuales se conservaron las relaciones isotópicas adquiridas durante su formación (Veizer et al., 1999; Jacobsen y Kaufman, 1999; Montañez et al., 2000; Melezhik et al., 2001; Jiang et al., 2007; Prokoph et al., 2008; Halverson et al., 2010; Zaky et al., 2019). Las variaciones de las razones isotópicas de ${ }^{87} \mathrm{Sr}{ }^{86} \mathrm{Sr}$ en rocas carbonáticas son las más utilizadas, en tanto que el uso de los isótopos del $\mathrm{O}$ y C está más restringido debido a que estos son afectados con mayor facilidad por la interacción con el agua meteórica y con fluidos intersticiales de alta temperatura (Fairchild et al., 1990). A pesar de la movilidad relativamente alta de los carbonatos durante los procesos de diagénesis y metamorfismo, en determinadas condiciones estas rocas pueden comportarse como un sistema cerrado (e.g., microambientes diagenéticos y/o metamórficos en donde no hay interacción con fases fluidas externas al sistema) y conservar sus improntas geoquímicas originales, permitiendo determinar la edad de sedimentación de los protolitos carbonáticos de complejos metamórficos, aún en condiciones de grado medio a alto (Brand y Veizer, 1980; Melezhik et al., 2001).

El Macizo Norpatagónico (MNP), ubicado en el sector norte de la Patagonia argentina, está conformado por un basamento ígneo-metamórfico Paleozoico a Triásico Inferior, el cual se encuentra cubierto por depósitos piroclásticos, volcánicos y sedimentarios mesozoicos-cenozoicos. Ha sido motivo de numerosos estudios en los últimos 15 años (e.g., Martínez Dopico et al., 2011; Rapalini et al., 2013; Ramos y Naipauer, 2014; Hervé et al., 2016, 2018; Rapela y Pankhurst, 2020; Serra Varela et al., 2020; González et al., 2021; entre otros), y cuenta con abundantes dataciones radiométricas, fundamentalmente sobre las rocas ígneas y, en menor medida, en las rocas metasedimentarias que lo componen. Estas dataciones han permitido conocer edades de cristalización y metamorfismo, como así también las edades máximas de depositación y proveniencia de los protolitos sedimentarios. Sin embargo, las rocas carbonáticas han sido poco estudiadas como un acotador de la edad de sedimentación debido a que los afloramientos son muy escasos, y han sido mayormente vinculadas al Ediacariano-Cámbrico medio (e.g., López de Luchi et al., 2010; González et al., 2011; Varela et al., 2014).

En la región central de la provincia de Chubut se expone una secuencia de mármoles conocida

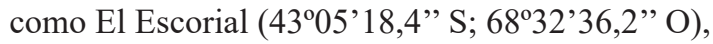
los que han sido descritos como integrantes de la Formación Cushamen (Miembro El Escorial, Proserpio, 1987; Murra et al., 2017), y corresponden a los afloramientos más orientales de esta unidad geológica (Figuras 1A y B).

La Formación Cushamen (Volkheimer, 1964) está compuesta por una secuencia de rocas paraderivadas metamorfizadas en condiciones de bajo grado (filitas y metapsammitas), esquistos micáceos y cuarzomicáceos y metacuarcitas que localmente gradan a gneises y migmatitas. Recientemente, Serra-Varela et al. (2020) han propuesto renombrar esta unidad litoestratigráfica como Complejo Metamórfico Cushamen (CMC), en función de su alta complejidad debido a su gran variedad litológica, fuerte deformación y metamorfismo, por lo que en el presente trabajo se adoptará la denominación sugerida por estos autores.

La edad máxima de sedimentación de los protolitos del CMC ha sido restringida al Devónico Medio a partir de edades herencia en circones detríticos (ca. $385 \mathrm{Ma}$; e.g., Hervé et al., 2005, 2018), mientras que el metamorfismo de esta unidad estaría acotado por el emplazamiento de granitoides no deformados del Pérmico temprano de la Formación Mamil Choique, datados en 286$261 \mathrm{Ma}$ (Dalla Salda et al., 1994; Cerredo, 1997; Cerredo y López de Luchi, 1998; Duhart et al., 2002; Giacosa et al., 2004; Lucassen et al., 2004; Varela et al., 2005; Pankhurst et al., 2006, 2014; López de Luchi et al., 2011; Renda et al., 2021). De esta manera, la edad de sedimentación del CMC está pobremente definida entre 385-286 Ma.

La presente contribución aporta nuevos datos que permiten acortar con mayor precisión la edad de sedimentación y metamorfismo del CMC. En este sentido, el trabajo da a conocer los primeros valores isotópicos de $\mathrm{Sr}$ de los mármoles correspondientes a la Unidad El Escorial del CMC, e indica la posibilidad de la existencia de una plataforma mixta hacia el suroeste del Gondwana suroccidental entre el Devónico tardío y el Carbonífero temprano. También se presenta la edad del metamorfismo del CMC en la región centro-sur del MNP, obtenida 


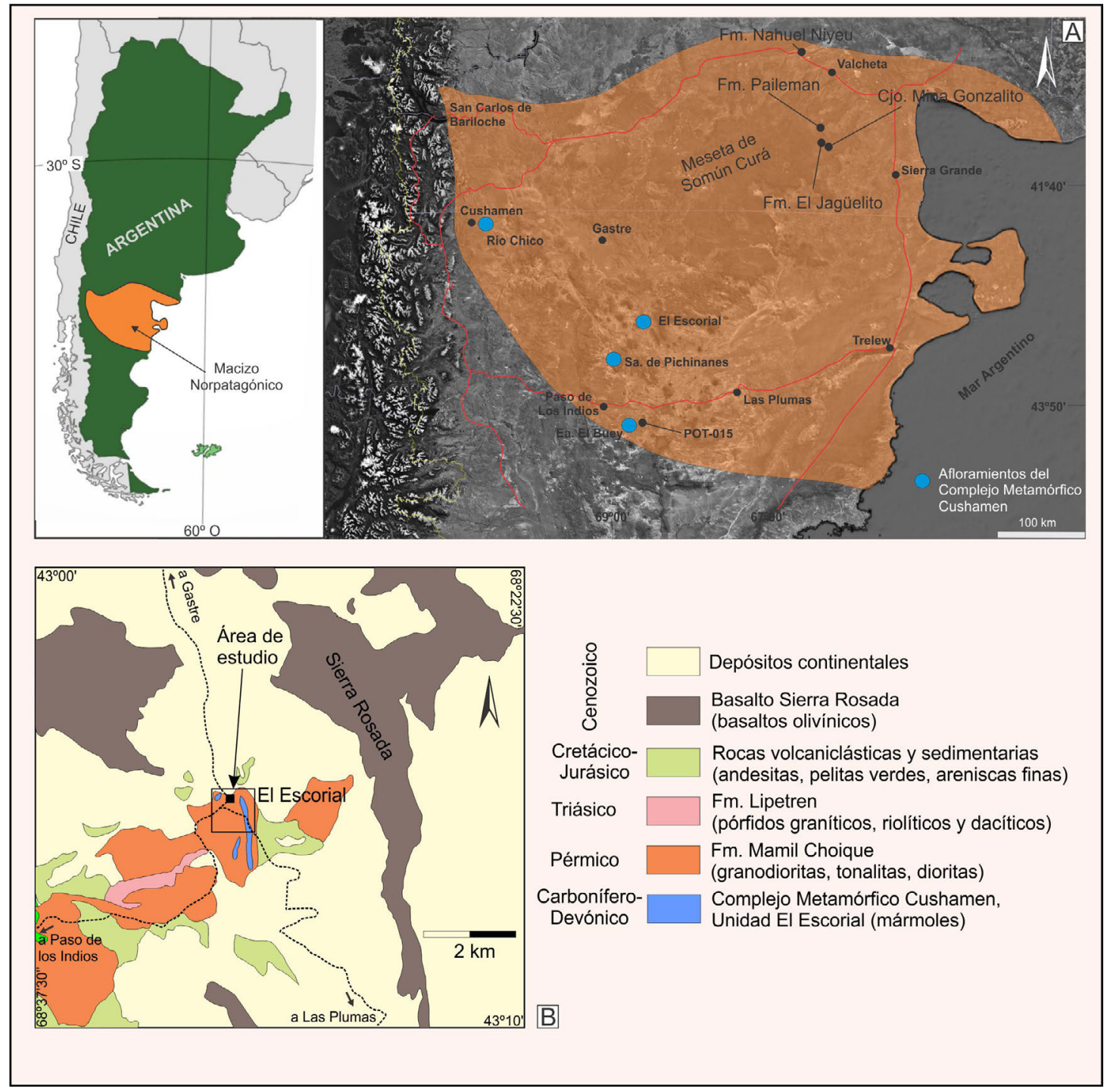

FIG. 1. A. Ubicación de los principales afloramientos del Complejo Metamórfico Cushamen en el contexto del Macizo Norpatagónico y de otros afloramientos de rocas carbonáticas. B. Mapa geológico del área de El Escorial, tomado de Anselmi et al. (2004).

mediante el método de datación CHIME (Chemical Th-U-total $\mathrm{Pb}$ Isochron Method) en monacitas de gneises migmáticos.

\section{Marco geológico}

El basamento premesozoico de la Patagonia extrandina está subdividido en el MNP al norte y el Macizo del Deseado hacia el sur. El límite norte del MNP con el margen suroccidental de Gondwana se encuentra cubierto por la cuenca mesozoica-cenozoica del Colorado (Lesta et al., 1980). En este sector nororiental del MNP, las unidades del basamento son rocas metasedimentarias del Paleozoico inferior (530 Ma, Formación Jagüelito; 513 Ma, Formación Nahuel Niyeu; 475 Ma, Formación Mina Gonzalito), intruidas por unidades ígneas con edades comprendidas entre los 545 y $246 \mathrm{Ma}$ (Pankhurst et al., 2006; Rapalini et al., 2013; Greco et al., 2015; González et al., 2021 y referencias allí citadas). La meseta de Somún Curá (Fig. 1A), compuesta por rocas y sedimentos del Mesozoico-Cenozoico, divide 
esta región del sector suroccidental del MNP, cuyo basamento metamórfico está compuesto por rocas metasedimentarias del Paleozoico medio (CMC: $362 \pm 10$ Ma y $371 \pm 33$ Ma, Ostera et al., 2001; 335$286 \mathrm{Ma}$, Hervé et al., 2005; 329 Ma, Gneis El Maitén, Pankhurst et al., 2006) intruidas por unidades ígneas de edad carbonífera a triásica.

En el MNP, entre los pocos estudios sobre las unidades metacarbonáticas existentes, se puede citar a Varela et al. (2014), quienes utilizan quimioestratigrafía de Sr sobre las calizas de la Formación El Jagüelito y sobre los mármoles de bajo grado del Complejo Mina Gonzalito y la Formación Pailemán (Fig. 1A), atribuyéndoles edades de sedimentación del Ediacariano medio a superior al Cámbrico inferior. En el sector noreste del MNP, González et al. (2011) han reconocido bloques de calizas dentro de metaconglomerados (Formación El Jagüelito) a las que le asignan una edad cámbrica inferior sobre la base de la presencia de arqueociátidos pobremente preservados. En la región de Valcheta (Fig. 1A), López de Luchi et al. (2010) mencionan la presencia de esporádicos niveles de mármoles y anfibolitas como integrantes de la Formación Nahuel Niyeu del Cámbrico tardío a medio (Fig. 1A). Hacia el sector centro y sur del MNP, Proserpio (1987) señala afloramientos de "calizas cristalinas" de reducidas dimensiones en las cercanías de Paso de Indios, y hace referencia también a otros autores que las mencionan en sus respectivos estudios (ver referencias en Proserpio, 1987). Según este autor, la distribución de los afloramientos de mármoles consiste en tres cuerpos que originariamente formaban uno solo, y que se desmembraron debido a un fallamiento moderno. Por otra parte indica que, hacia el sector sur de El Escorial, se encuentran enclaves metamórficos de hasta $1 \mathrm{~km}$ de largo de metapsammitas, incluidos en una masa granodiorítica-tonalítica, con una foliación penetrativa importante y compuestos por cuarzo-feldespato-clorita-mica blanca de grano fino \pm epidoto \pm apatita \pm circón.

Dentro de este marco geológico se han propuesto algunas hipótesis sobre la evolución geodinámica de la Patagonia extrandina, en las se propone un origen parautóctono, en donde la Patagonia se adosó al borde suroccidental de Gondwana a finales del Paleozoico (e.g., Ramos, 2008); mientras que otras se inclinan por un origen autóctono, con el MNP como parte de Gondwana en tiempos precarboníferos, según evidencias geocronológicas e isotópicas, que indicarían que el magmatismo del extremo nororiental del MNP tiene características similares al generado en los orógenos Pampeano y Famatiniano, por lo que se concluye que representa la extensión de estos ciclos orogénicos hacia el sur (Dalla Salda et al., 1991; Rapalini et al., 2010, 2013; Pankhurst et al., 2006; Martínez Dopico et al., 2011; Greco et al., 2015, 2017; Heredia et al., 2018). En este último modelo, Pankhurst et al. (2006) proponen que la región al sur del MNP (el Macizo del Deseado) se adosa al Gondwana suroccidental, genera hacia los $335 \mathrm{Ma}$ una subducción con vergencia al noreste (por debajo del MNP), con aporte de sedimentos desde el norte (coordenadas actuales) y, posteriormente, origina el Complejo Metamórfico Cushamen.

\section{Muestreo y técnicas analíticas}

\subsection{Análisis isotópicos de mármoles}

Se recolectaron 37 muestras, de las cuales 25 fueron seleccionadas para petrografía y 10 para análisis químicos e isotópicos. Para estimar inicialmente la cantidad de calcita y dolomita, se cortaron losas $(6 \times 3 \times 1 \mathrm{~cm})$ y la superficie se tiñó con alizarina roja $\mathrm{S}$ $(0,3 \mathrm{~g}$ de alizarina en $100 \mathrm{ml}$ de solución de $\mathrm{HCl}$ al $1,5 \%$ ) durante 4 minutos, y luego se lavó suavemente con agua destilada. El porcentaje de calcita manchada en la superficie de la losa se determinó mediante imágenes por ordenador (utilizando el programa Image-J 1.48v, Rasband, 2017). Se seleccionaron muestras con calcita $>90 \%$ en su mayoría $(n=14)$ (Tabla 1). Luego fueron analizados $\mathrm{Ca}, \mathrm{Mg}$ y $\mathrm{Mn}, \mathrm{y}$ se determinó el residuo insoluble (R.I.). Las muestras con bajos valores de R.I. se sometieron a un cribado químico de acuerdo con las relaciones $\mathrm{Mn} / \mathrm{Sr}$ y $\mathrm{Mg} / \mathrm{Ca}$ (Brand y Veizer, 1980; Melezhik et al., $2001 \mathrm{y}$ referencias allí citadas) para elegir aquellas que no habrían sufrido un impacto significativo de alteraciones postsedimentarias (Tabla 2). Los análisis del Sr se realizaron en el Centro de Geoquímica Isotópica y Geocronología de la Universidad Complutense de Madrid. Para excluir la contaminación de otros minerales, las muestras de carbonato $(30 \mathrm{mg})$ fueron lixiviadas en una solución de ácido acético al 10\% y luego centrifugadas para eliminar el residuo insoluble (Fuenlabrada y Galindo, 2001). Además, se determinó el residuo insoluble (R.I. en \% en peso). La solución se evaporó posteriormente y luego se disolvió en $3 \mathrm{ml}$ de $\mathrm{HCl}$ 2,5 N. El Sr se separó por 
TABLA 1. UBICACIÓN, DESCRIPCIÓN DE CAMPO Y PETROGRAFÍA DE MUESTRAS DE LOS MÁRMOLES EL ESCORIAL Y LA ROCA DE CAJA.

\begin{tabular}{|c|c|c|c|c|c|}
\hline Muestra & latitud & longitud & Descripción & $\begin{array}{l}\text { \% calcita } \\
\text { (teñido) }\end{array}$ & $\begin{array}{c}\text { Mineralogía } \\
\text { (abreviación mineral según } \\
\text { Whitney y Evans, 2010) } \\
\end{array}$ \\
\hline MNP-28O21* & $43^{\circ} 05^{\prime} 17,6^{\prime \prime}$ & $68^{\circ} 32^{\prime} 26,3^{\prime \prime}$ & anfibolita en contacto con mármol & - & Am-Pl-Qz-Bt \\
\hline MNP-28O22* & & & aplita, rosada, grano muy fino (<1 mm) & - & Qz-Kfs-Ms \\
\hline MNP-28O23* & & & granodiorita grano grueso (1-5 mm) & - & Qz-Pl-Bt $\pm \mathrm{kfs}$ \\
\hline MNP-28O24* & & & mármol gris claro, grano medio, homogéneo & 91,56 & Cal-Srp-Opq-Ol \\
\hline MNP-28O25* & & & mármol gris claro, grano fino, bandeado & 89,56 & Cal-Qz-Opq-Di-Phl \\
\hline MNP-28O26* & & & mármol blanco, grano fino, homogéneo & 91,81 & Cal-Qz-Opq \\
\hline MNP-28O27* & & & mármol gris claro a blanco, grano fino, bandeado & 76,98 & Cal- Qz-Opq-Phl \\
\hline MNP-28O28* & & & mármol gris, grano fino, bandeado & 71,51 & Cal-Qz-Opq-Phl \\
\hline MNP-28O29* & & & mármol celeste, grano grueso, homogéneo & 84,81 & Cal-Qz-Phl \\
\hline MNP-28O30* & & & mármol gris, grano fino, bandeado & 76,81 & $\mathrm{Cal} \pm \mathrm{Phl}-\mathrm{Qz}$ \\
\hline MNP-28O31* & & & mármol gris, grano grueso, bandeado & 79,52 & Cal-Qz-Opq-Di-Phl \\
\hline MNP-28O32* & & & anfibolita en contacto con mármol & - & Am-Pl-Qz-Bt \\
\hline MNP-28O33* & & & granodiorita grano grueso $(1-5 \mathrm{~mm})$ & - & Qz-Pl-Bt \pm kfs \\
\hline MNP-28O34-2 & $43^{\circ} 05^{\prime} 17,6^{\prime \prime}$ & $68^{\circ} 32^{\prime} 26,3^{\prime \prime}$ & mármol gris, grano fino, homogéneo & 98,56 & $\mathrm{Cal} \pm \mathrm{Phl}$ \\
\hline MNP-28O34-3 & & & mármol blanco, grano medio, masivo, deformado & 98,43 & $\mathrm{Cal} \pm \mathrm{Phl}-\mathrm{Qz}$ \\
\hline MNP-28O34-4 & & & mármol blanco, grano medio, homogéneo & 98,66 & $\mathrm{Cal} \pm \mathrm{Phl}-\mathrm{Qz}$ \\
\hline MNP-28O34-5a & & & mármol blanco, grano fino, homogéneo & 95,31 & $\mathrm{Cal} \pm \mathrm{Phl}-\mathrm{Qz}$ \\
\hline MNP-28O34-5b & & & mármol blanco, grano fino, bandeado & 95,21 & $\mathrm{Cal} \pm \mathrm{Phl}-\mathrm{Qz}$ \\
\hline MNP-28O34-6* & & & mármol celeste, grano grueso, homogéneo & 14,64 & Cal-Qz-Opq-Phl \\
\hline MNP-28O34-8 & & & mármol gris, grano medio, homogéneo & 78,04 & Cal-Srp-Opq-Ol \\
\hline MNP-28O34-9a & & & mármol blanco, grano medio, homogéneo & 85,36 & Cal-Qz-Opq \\
\hline MNP-28O34-9b & & & mármol blanco, grano medio, bandeado & 91,88 & Cal-Qz-Phl \\
\hline MNP-28O34-10 & & & mármol blanco, grano fino, bandeado & 97,45 & Cal-Qz-Opq \\
\hline MNP-28O34-11 & $43^{\circ} 05^{\prime} 12,6$ ' & $68^{\circ} 32^{\prime} 35,5^{\prime \prime}$ & mármol blanco, grano medio, homogéneo & 98,07 & Cal-Qz-Opq-Grt \\
\hline MNP-28O34-12a & $43^{\circ} 05^{\prime} 10,1$ ' & $68^{\circ} 32^{\prime} 40,4^{\prime \prime}$ & mármol celeste, grano fino, homogéneo & 86,01 & Cal-Qz-Opq-Di-Phl \\
\hline MNP-28O34-12b & & & mármol gris, grano grueso, homogéneo & 95,75 & Cal-Qz-Opq-Di-Phl \\
\hline MNP-28O35-15 & $43^{\circ} 05^{\prime} 35,1^{\prime \prime}$ & $68^{\circ} 32^{\prime} 08,2^{\prime \prime}$ & mármol gris, grano medio, homogéneo & 97,29 & Cal-Opq \\
\hline MNP-28O35-16* & & & mármol gris claro, grano grueso, homogéneo & 54,61 & Cal-Opq \\
\hline MNP-28O35-17a* & & & mármol gris, grano fino, bandeado & 96,23 & Cal-Opq-Grt \\
\hline MNP-28O35-17b & $43^{\circ} 05^{\prime} 34,5^{\prime \prime}$ & $68^{\circ} 32^{\prime} 12,2^{\prime \prime}$ & mármol blanco, grano fino, homogéneo & 97,81 & Cal-Opq-Grt \\
\hline
\end{tabular}

* No analizado para geoquímica. 
TABLA 2. CONTENIDO DE CA, MG, MN Y RB, Y COMPOSICIÓN ISOTÓPICA DEL SR DE LOS MÁRMOLES DE EL ESCORIAL.

\begin{tabular}{|c|c|c|c|c|c|c|c|c|c|c|}
\hline Muestra & Ubicación & $\begin{array}{c}\mathrm{Sr} \\
(\mathbf{p p m})\end{array}$ & ${ }^{87} \mathrm{Sr} /{ }^{86} \mathrm{Sr}$ & $\begin{array}{c}\text { Ca } \% \\
\text { peso }\end{array}$ & $\begin{array}{c}\text { Mg \% } \\
\text { peso }\end{array}$ & $\begin{array}{c}\mathbf{R b} \\
(\mathbf{p p m})\end{array}$ & $\begin{array}{c}\text { Mn } \\
(\mathbf{p p m})\end{array}$ & $\mathrm{Mg} / \mathrm{Ca}$ & $\mathrm{Mn} / \mathrm{Sr}$ & R.I. \% \\
\hline MNP-28034-2 & \multirow{12}{*}{$\begin{array}{c}\text { Banco } \\
\text { La Cruz }\end{array}$} & 325 & 0,707936 & 45,55 & 0,21 & 3 & 210 & 0,00461 & 0,64615 & 2,50 \\
\hline MNP-28034-3 & & 513 & 0,708183 & 46,21 & 0,22 & 2 & 38 & 0,00432 & 0,07407 & 0,93 \\
\hline MNP-28034-4 & & 528 & 0,708214 & 46,02 & 0,33 & 2 & 59 & 0,00717 & 0,11174 & 1,63 \\
\hline MNP-28034-5a & & 242 & 0,707757 & 45,49 & 0,25 & 1 & 107 & 0,00549 & 0,44214 & 2,31 \\
\hline MNP-28034-5b & & 242 & - & 44,81 & 0,49 & 1 & 66 & 0,01093 & 0,27272 & 2,46 \\
\hline MNP-28034-8 & & 438 & - & 41,31 & 3,41 & 1 & 10 & 0,08231 & 0,02283 & 0,86 \\
\hline MNP-28034-9a & & 427 & - & 42,44 & 1,51 & 2 & 162 & 0,03534 & 0,37939 & 2,31 \\
\hline MNP-28034-9b & & 427 & 0,707681 & 44,71 & 0,38 & 2 & 129 & 0,00851 & 0,30211 & 2,09 \\
\hline MNP-28034-10 & & 270 & 0,707923 & 45,56 & 0,31 & 1 & 89 & 0,00658 & 0,32962 & 1,47 \\
\hline MNP-28034-11 & & 789 & 0,708198 & 45,86 & 0,24 & 3 & 34 & 0,00523 & 0,04309 & 0,39 \\
\hline MNP-28034-12a & & 379 & - & 41,69 & 2,09 & 2 & 165 & 0,05013 & 0,43535 & 2,18 \\
\hline MNP-28034-12b & & 589 & 0,708249 & 43,84 & 1,77 & 2 & 14 & 0,040374 & 0,02376 & 1,12 \\
\hline MNP-28035-15 & \multirow{2}{*}{$\begin{array}{c}\text { Banco } \\
\text { La Escuela }\end{array}$} & 423 & 0,707875 & 45,31 & 0,32 & 1 & 143 & 0,00706 & 0,33806 & 2,29 \\
\hline MNP-28035-17b & & 728 & 0,708228 & 45,15 & 0,28 & 2 & 119 & 0,00621 & 0,16346 & 1,48 \\
\hline
\end{tabular}

R.I. \% peso: residuo insoluble.

medio de columnas de intercambio catiónico rellenas con resina BioRad ${ }^{\circledR} 50 \mathrm{~W}$ X12 (malla 200/400). El blanco de procedimiento era inferior a $2 \mathrm{ng}$ para Sr. La composición isotópica de $\mathrm{Sr}$ se determinó en un espectrómetro automatizado Multicollector SECTOR $54^{\circledR}$ y los valores de ${ }^{87} \mathrm{Sr}{ }^{86} \mathrm{Sr}$ se normalizaron a un valor ${ }^{86} \mathrm{Sr} /{ }^{88} \mathrm{Sr}$ de 0,1194 . La norma NBS-987 se analizó rutinariamente junto con las muestras del estudio y dio un valor medio de ${ }^{87} \mathrm{Sr} /{ }^{86} \mathrm{Sr}$ de $0,710251 \pm 0,00002(2 \sigma, \mathrm{n}=7)$. La incertidumbre analítica general se estima en $\pm 0,01 \%$. Para el $\mathrm{Rb}$, las muestras se hicieron reaccionar con ácido acético $0,5 \mathrm{M}$, se centrifugaron a $4.000 \mathrm{rpm}$ y se recogió el lixiviado para su análisis. El residuo se lavó dos veces con agua Millipore centrifugada y se añadió el nuevo lixiviado al primero. Posteriormente se secó y se volvió a disolver con $1 \mathrm{ml}$ de $\mathrm{HNO}_{3} 0,1 \mathrm{M}$ para análisis por ICP-MS. Además de los datos químicos obtenidos, se realizó la petrografía de 16 muestras de mármoles y de las respectivas rocas encajonantes de cada uno de los afloramientos (otras 7 muestras).

\subsection{Geocronología en monacita}

La edad del metamorfismo fue determinada en una muestra de gneis (POT-015) del Complejo
Metamórfico Cushamen, mediante el método CHIME (Chemical Th-U-total Pb Isochron Method o datación EPMA) en monacitas, el cual se basa en las concentraciones de $\mathrm{U}, \mathrm{Th}, \mathrm{Pb}$ y Tierra Raras (REE). El análisis de las monacitas se realizó con una microsonda electrónica SX-100 de la Universidad de Oviedo (España) bajo condiciones de $25 \mathrm{kv}$ y $100 \mathrm{nA}$, siguiendo los protocolos de Scherrer et al. (2000). Las edades obtenidas fueron corroboradas con el estándar de monacita IGME $6883 \mathrm{~A}$ (1.083 $\pm 1,5 \mathrm{Ma}, \mathrm{U}-\mathrm{Pb}$ ID-TIMS; EMPA $1.079 \pm 6 \mathrm{Ma})$. Se obtuvieron 53 análisis puntuales. El cálculo de la edad se efectuó según la propuesta de Williams et al. (1999) y Montel et al. (1996). La edad media ponderada $(2 \sigma)$ se obtuvo mediante Isoplot 4.15 (Ludwig, 2003). Para más detalles sobre el tratamiento de datos y cálculo de edad ver Fernández González et al. (2009).

\section{Resultados}

\subsection{Mármoles EI Escorial}

Corresponden a afloramientos restringidos a una franja lentiforme, de unos $3 \mathrm{~km}$ de largo por 100 a $300 \mathrm{~m}$ de espesor y con una orientación general 
N-S (Fig. 1B), asociados generalmente a pequeños lentes de anfibolitas que forman septos dentro de granodioritas, tonalitas y filones aplíticos de espesores no mayores a $15-20 \mathrm{~cm}$ (Fig. 2). Los mármoles configuran bancos heterogéneos con variaciones en el tamaño del grano $(0,5$ a $3 \mathrm{~mm})$, de las tonalidades (blancos, celestes, grises y rosados) y de las estructuras internas (macizos a levemente bandeados y plegados). La foliación principal observada en los bancos es de $315^{\circ}-320^{\circ} / 50^{\circ} \mathrm{NE}$, visible fundamentalmente en el bandeado composicional (Fig. 3).

En su mayoría, los mármoles son de composición calcítica (Tabla 1) con contenidos menores de dolomita \pm flogopita \pm cuarzo \pm diópsido \pm serpentina \pm granate \pm grafito. Petrográficamente, las rocas tienen una textura granoblástica de grano medio a fino ( $\sim, 1$ a $2 \mathrm{~mm})$. En general, se presentan como mosaicos granoblásticos de calcita poligonal, con

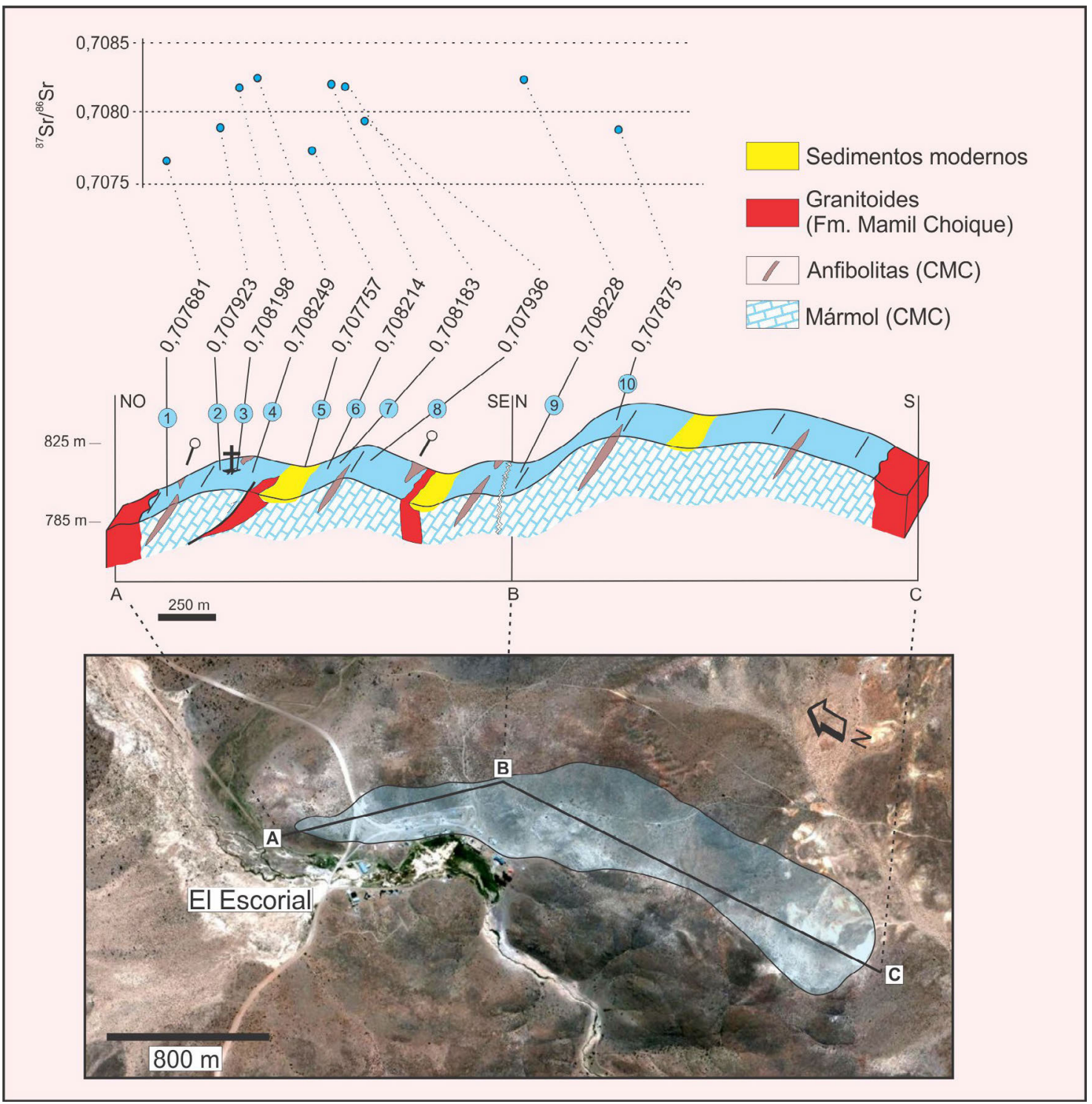

FIG. 2. Perfil esquemático de la sección estudiada con la ubicación de las muestras de mármol analizadas y el valor de sus razones isotópicas de ${ }^{87} \mathrm{Sr} /{ }^{86} \mathrm{Sr}$. Muestras: 1. MNP-28034-9b; 2. MNP-28034-10; 3. MNP-28034-11; 4. MNP-28034-12b; 5. MNP28034-5a; 6. MNP-28034-4; 7. MNP-28034-3; 8. MNP-28034-2; 9. MNP-28035-17b; 10. MNP-28035-15. 


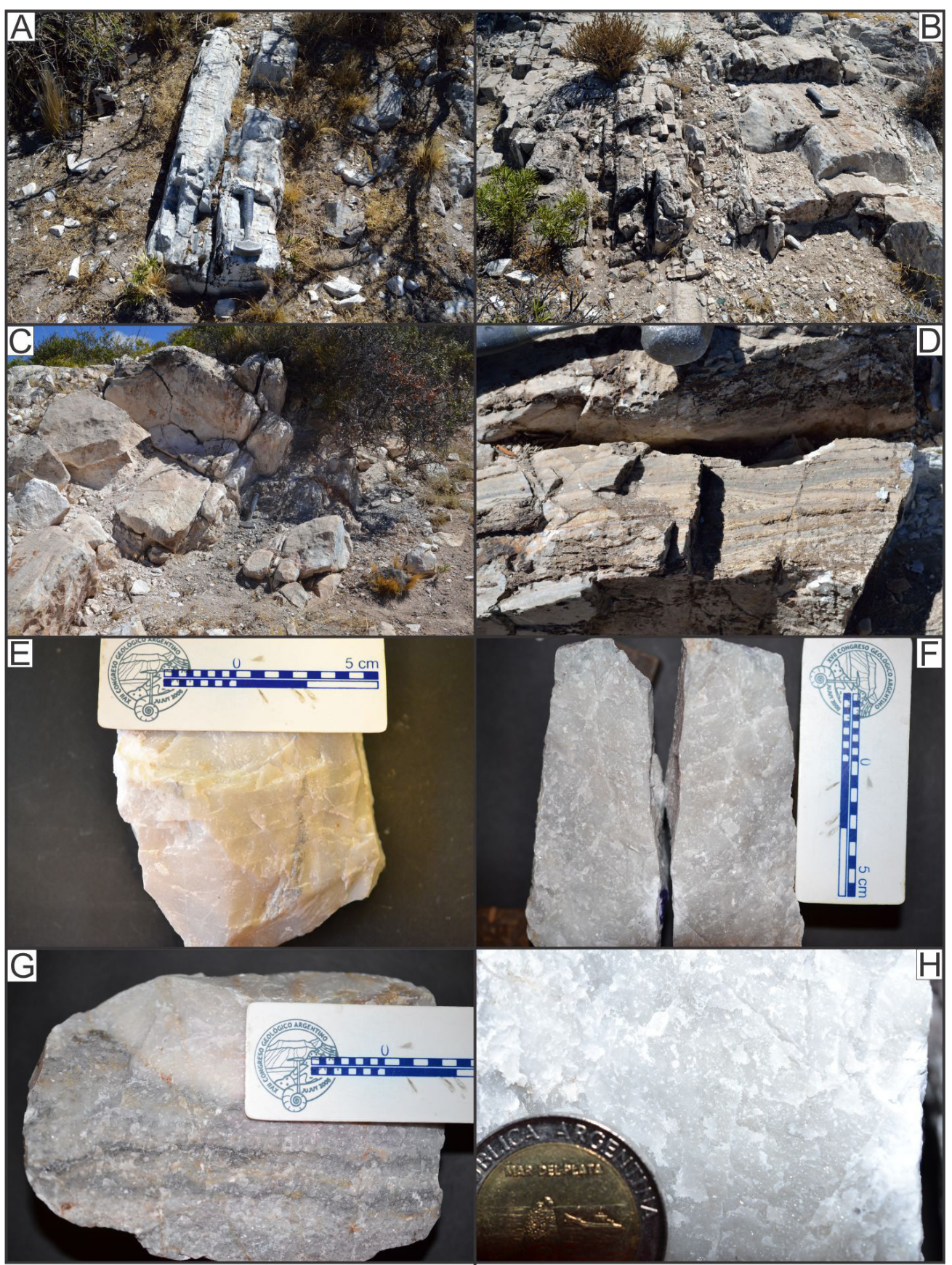

FIG. 3. A-D. Fotografías de los afloramientos más representativos de los mármoles de El Escorial. E-H. Fotografías de muestras macroscópicas mostrando algunas de las variedades de mármoles encontradas en los afloramientos.

tamaños de grano medio de 1 a 2 mm (Fig. 4A y B). Particularmente, hay sectores con un tamaño de grano mucho menor, entre 0,1 y $0,5 \mathrm{~mm}$ (Fig. 4C), y en ocasiones puntuales se observa leve deformación representada por la orientación de granos de calcita de la matriz que rodean a porfidoblastos de mayor tamaño, elongados y con extinción ondulante (Fig. 4D). Los minerales accesorios principales son diópsido, 


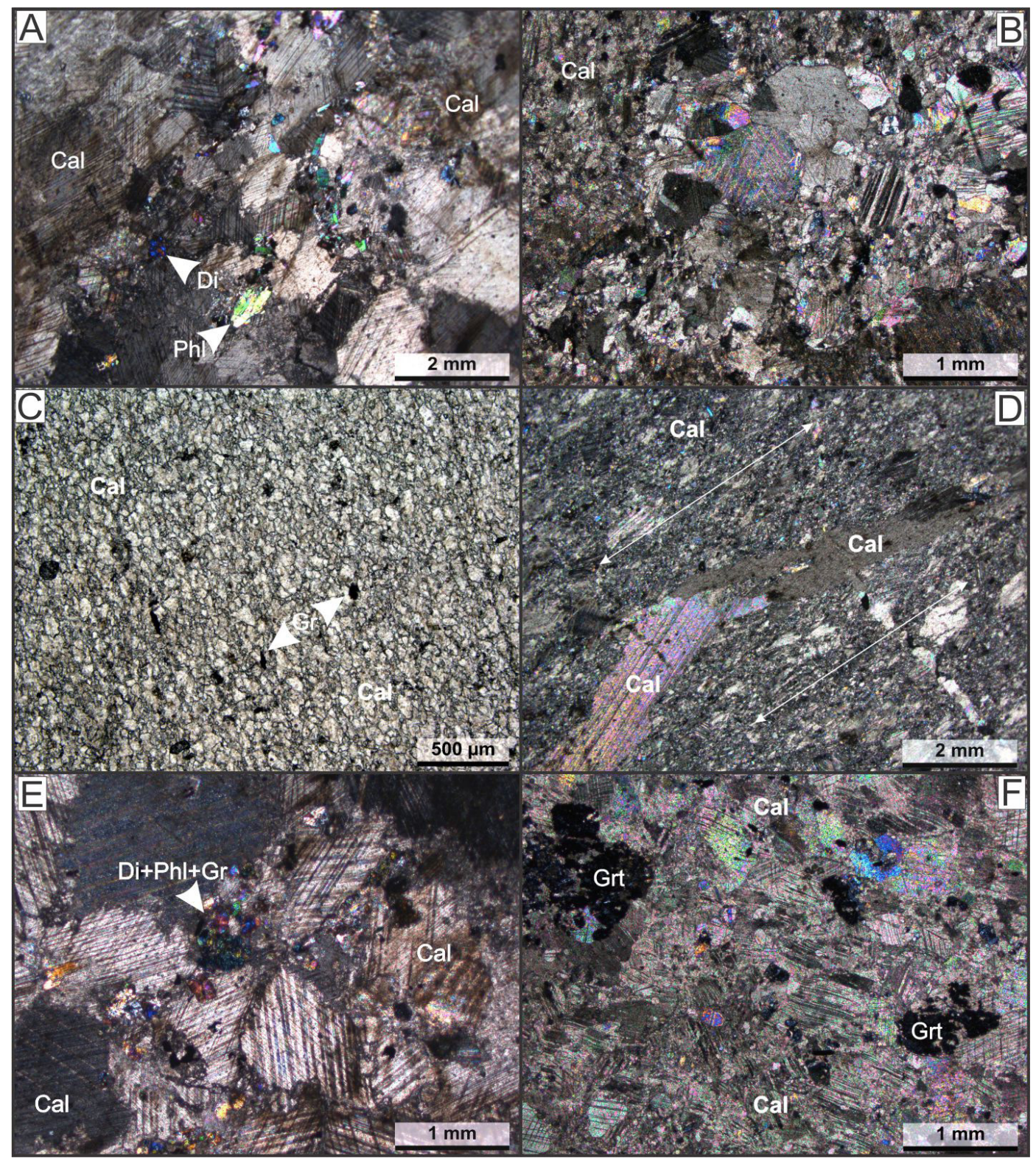

FIG. 4. Fotomicrografías de las muestras representativas de las variedades presentes en los afloramientos de mármoles. En D se indica la dirección de estiramiento de la calcita debido a deformación frágil-dúctil en sectores localizados. A. B. D. E. F. Nicoles cruzados. C. Nicoles paralelos. Abreviatura de minerales según Whitney y Evans (2010).

flogopita, granate y cuarzo en cantidades variables, lo que depende de los niveles que se analicen; por sectores las cantidades de minerales accesorios varían entre el 2 y $5 \%$ volumen modal, mientras que en bandas con mayores impurezas llegan al 15-20\%. En estos últimos, es común encontrar a modo de agregados o nódulos, la asociación diópsido-flogopita-grafito
(Fig. 4E). Diópsido y flogopita se localizan, además, en blastos individuales suhedrales a anhedrales de tamaños entre 0,7-0,3 mm (Fig. 4A). El granate es esporádico $(<2 \%)$ : en las variedades de grano fino, se lo observa de manera anhedral en el mosaico poligonal calcítico (Fig. 4C) y en las de grano medio, con formas subhedrales desmembradas (Fig. 4F). 
La paragénesis calcita+flogopita+diópsido+granate, registrada en los niveles impuros, indica que estas rocas alcanzaron condiciones de grado medio a alto de metamorfismo.

Desde el punto de vista químico (Tabla 2), los mármoles analizados son de composición cálcica, con porcentajes modales de calcita superiores al 95\% y bajos porcentajes de residuos insolubles (R.I.: 0,5 a 2,5\% peso). Del total de 14 muestras, 4 fueron descartadas por posibles alteraciones postsedimentarias atendiendo a sus valores elevados de las razones $\mathrm{Mn} / \mathrm{Sr}(0,02-0,64)$ y $\mathrm{Mg} / \mathrm{Ca}(0,004-$ 0,082). Los valores de $\mathrm{Rb}$ son todos inferiores a $3 \mathrm{ppm}$, lo cual es un indicador de que no se ha formado $\mathrm{Sr}$ radiogénico durante procesos postsedimentarios. En las 10 muestras restantes se determinó la razón ${ }^{87} \mathrm{Sr} /{ }^{86} \mathrm{Sr}$ cuyos valores varían entre 0,70768 y 0,70825 (Fig. 5, Tabla 2).

\subsection{Edad del metamorfismo en el gneis (POT- 015) del CMC}

La muestra analizada (POT-015) es un gneis bandeado con alternancia de niveles melano- y leucocráticos granoblásticos. Ella fue extraída en el

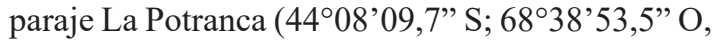
Fig. 1A), a $120 \mathrm{~km}$ al sur de los mármoles El Escorial. Son afloramientos discontinuos, de dimensiones reducidas, fuertemente alterados y observables en pequeñas ventanas erosivas de la cubierta sedimentaria moderna, que exhiben una foliación principal con orientación $\mathrm{N} 45-57^{\circ}$ y buzamientos de $60-70^{\circ} \mathrm{SE}$. Estas unidades de basamento metamórfico, al igual que los mármoles El Escorial, forman septos incluidos en granitos postmetamórficos de la Formación Mamil Choique (289 \pm 2 Ma; Pankhurst et al., 2006).

La mineralogía del gneis está representada por cuarzo, plagioclasa, feldespato potásico, granate, biotita, cordierita y escasa sillimanita y los minerales accesorios lo están por circón, apatita, monacita e ilmenita. La muscovita está presente como mineral de alteración. Posibles pseudomorfos de clorita+biotita según ortopiroxeno son reconocidos en esta muestra. La presencia de texturas de fusión parcial y posible asociación mineral con ortopiroxeno sugiere condiciones en facies de granulitas para el pico metamórfico.

La monacita se encuentra como inclusión en granate y en la matriz de la roca. Son granos subredondeados, poco fracturados y con tamaños de entre 20 y $30 \mu \mathrm{m}$. Poseen una composición relativamente homogénea con tenores promedio $(\mathrm{n}=49)$ de $\mathrm{Ce}_{2} \mathrm{O}_{3}=27,21 \%(25,46-29,53), \mathrm{Y}_{2} \mathrm{O}_{3}=2,33 \%$ $(0,33-4,68), \Sigma_{\text {Tierras Raras restantes }}=33,23 \%(31,9-34,1)$, $\mathrm{UO}_{2}=0,66 \%(0,31-0,96), \mathrm{ThO}_{2}=4,56 \%(3,16-5,42)$ y $\mathrm{PbO}=0,09 \%(0,05-0,15)$ (Tabla 3 ).

La edad calculada a partir de 49 análisis es de $311 \pm 27 \mathrm{Ma}$, con MSWD=0,40 (2 $\sigma$, Fig. 6). De los 53 estudios realizados, cuatro fueron descartados. Tres de ellos tienen alto contenido de $\mathrm{Al}_{2} \mathrm{O}_{3}$ o $\mathrm{SiO}_{2}$ indicio de posible contaminación con la matriz, mientras que el otro fue eliminado por tener una

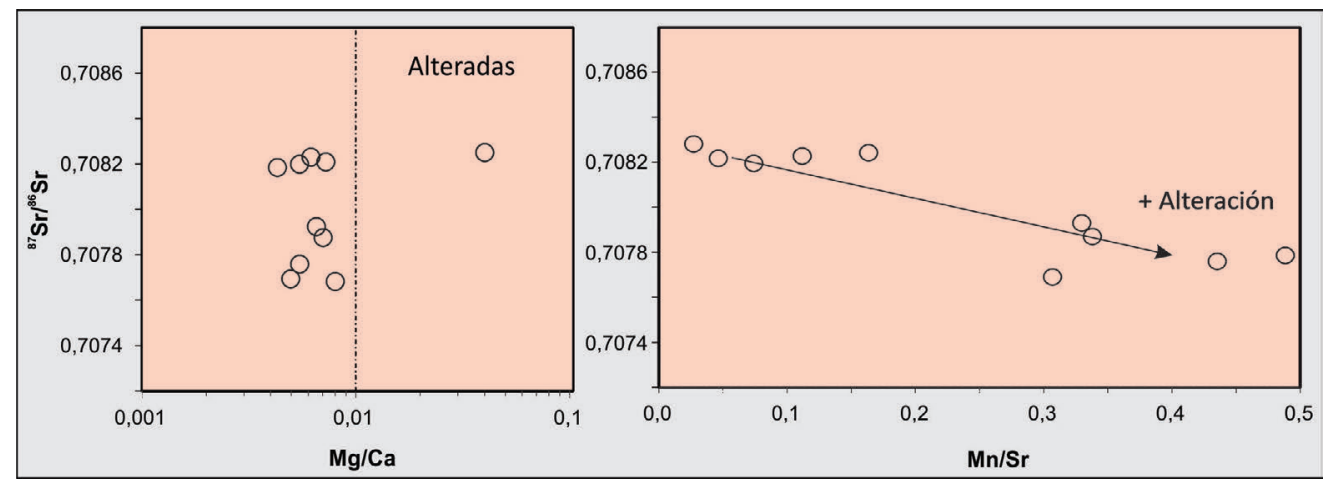

FIG. 5. Gráficos de cribado químico de los mármoles de El Escorial que muestran los límites de alteración pos-deposicionales de acuerdo con Melezhik et al. (2001). La diagénesis y el metamorfismo tienden a empobrecer a las rocas carbonáticas en $\mathrm{Sr}$, y a enriquecerlas en $\mathrm{Mn}$, y en consecuencia las relaciones $\mathrm{Mg} / \mathrm{Ca}$ y $\mathrm{Mn} / \mathrm{Sr}$ son los principales indicadores del grado de modificación sufrido por estas rocas. Además, es importante recalcar la escasa concentración de Rb, indicativo de un nulo o muy bajo aporte de Sr radiogénico proveniente de su desintegración. 


\begin{tabular}{|c|c|c|c|c|c|c|c|c|c|c|c|c|c|c|c|c|c|c|c|c|c|c|}
\hline $\begin{array}{l}\text { Puntos } \\
\text { de } \\
\text { análisis }\end{array}$ & $\mathrm{P}_{2} \mathrm{O}_{5}$ & $\mathrm{I}_{2} \mathrm{O}_{3}$ & $\mathrm{O}_{2}$ & $\mathrm{aO}$ & $Y_{2} \mathbf{O}_{3}$ & $\mathrm{e}_{2} \mathrm{O}_{3}$ & $\mathrm{r}_{2} \mathbf{O}_{3}$ & $\mathbf{y}_{2} \mathbf{O}_{3}$ & $\mathrm{Ho}_{2} \mathrm{O}_{3}$ & $\mathrm{La}_{2} \mathrm{O}_{3}$ & $\mathrm{Nd}_{2} \mathrm{O}_{3}$ & $\mathrm{Sm}_{2} \mathrm{O}_{3}$ & $\mathrm{Gd}_{2} \mathrm{O}_{3}$ & $\mathbf{T b}_{2} \mathrm{O}_{3}$ & $\mathrm{Er}_{2} \mathrm{O}_{3}$ & $\mathbf{Y b}_{2} \mathrm{O}_{3}$ & PbO & $\mathrm{ThO}_{2}$ & $\mathbf{U O}_{2}$ & Total & $\begin{array}{l}\text { Edad } \\
\text { (Ma) }\end{array}$ & En \\
\hline \multicolumn{23}{|l|}{$\%$ peso } \\
\hline $1 / 1$ & 30,366 & 022 & 14 & 082 & 332 & 9,529 & 211 & 103 & 000 & 14,554 & 12,231 & 2,296 & 1,360 &, 044 & 0,011 & 0,000 & 0,075 & 4,682 & 0,605 & 100,716 & 267 & 96 \\
\hline $10 / 1$ & 31,130 & 0,063 & 0,331 & 1,237 & 2,717 & 26,385 & 2,934 & 0,741 & 0,038 & 14,228 & 11,226 & 2,107 & 1,876 & 0,182 & 0,136 & 0,000 & 0,095 & 4,648 & 0,686 & 100,760 & 327 & 93 \\
\hline $12 / 1$ & 31,162 & 0,012 & 0,071 & 1,324 & 3,436 & 25,991 & 2,954 & 0,829 & 0,037 & 13,401 & 11,437 & 2,068 & 1,681 & 0,171 & 0,203 & 0,028 & 0,081 & 4,963 & 0,762 & 100,609 & 258 & 85 \\
\hline $13 / 1$ & 31,137 & 0,009 & 0,019 & 1,371 & 2,433 & 26,742 & 2,989 & 0,628 & 0,020 & 13,874 & 11,508 & 2,042 & 1,557 & 0,130 & 0,133 & 0,000 & 0,096 & 4,917 & 0,917 & 100,520 & 287 & 81 \\
\hline $14 / 1$ & 31,087 & 0,065 & 0,307 & 1,067 & 1,484 & 27,578 & 3,093 & 0,422 & 0,001 & 14,080 & 12,069 & 2,188 & 1,536 & 0,104 & 0,071 & 0,000 & 0,080 & 5,111 & 0,535 & 100,880 & 277 & 91 \\
\hline $15 / 1$ & 31,022 & 0,020 & 0,043 & 1,170 & 2,768 & 27,056 & 2,940 & 0,648 & 0,031 & 13,716 & 11,381 & 2,049 & 1,615 & 0,163 & 0,146 & 0,003 & 0,089 & 4,424 & 0,734 & 100,017 & 309 & 95 \\
\hline $18 / 1$ & 31,276 & 0,018 & 0,229 & 1,001 & 2,982 & 27,075 & 3,080 & 0,585 & 0,045 & 13,142 & 12,013 & 2,156 & 1,593 & 0,129 & 0,145 & 0,014 & 0,082 & 4,728 & 0,325 & 100,617 & 334 & 111 \\
\hline $19 / 1$ & 31,704 & 0,015 & 0,235 & 1,113 & 0,594 & 27,894 & 3,118 & 0,209 & 0,000 & 14,430 & 12,320 & 2,245 & 1,487 & 0,082 & 0,026 & 0,000 & 0,084 & 4,789 & 0,580 & 100,923 & 299 & 96 \\
\hline $2 / 1$ & 30,215 & 0,003 & 144 & 1,059 & 1,143 & 28,769 &, 155 & 0,370 & 0,006 & 13,889 & 12,017 & 2,433 & 2,038 & 0,125 & 0,043 & 0,000 & 0,079 & 4,292 & 0,596 & 100,376 & 300 & 103 \\
\hline $20 / 1$ & 30,850 & 0,063 & 0,096 & 1,190 & 2,175 & 27,820 & 3,027 & 0,541 & 0,017 & 14,044 & 11,743 & 2,061 & 1,443 & 0,118 & 0,107 & 0,000 & 0,114 & 4,495 & 0,828 & 100,729 & 376 & 92 \\
\hline $21 / 1$ & 30,975 & 0,039 & 0,046 & 1,295 & 2,169 & 27,524 & דנינו & 0,517 & 0,030 & 14,037 & 11,164 & 1,947 & 1,389 & 0,119 & 0,109 & 0,000 & 0,126 & 4,512 & 0,952 & 99 & 1 & 88 \\
\hline $22 / 1$ & 31,211 & 0,012 & 0,412 & 0,998 & 1,453 & 27,709 & 3,140 & 0,270 & 0,004 & 13,994 & 12,522 & 2,194 & 1,348 & 0,072 & 0,053 & 0,000 & 0,076 & 4,898 & 0,452 & 100,819 & 283 & 100 \\
\hline $23 / 1$ & 30,781 & 0,169 & 0,339 & 1,049 & 2,082 & 27,109 & 3,064 & 0,498 & 0,024 & 14,016 & 12,022 & 2,108 & 1,519 & 0,113 & 0,107 & 0,000 & 0,065 & 5,004 & 0,528 & 100,598 & 231 & 94 \\
\hline $24 / 1$ & 30,927 & 0,000 & 0,157 & 1,142 & 1,906 & 27,874 & 3,088 & 0,510 & 0,000 & 13,999 & 11,966 & 2,127 & 1,493 & 0,124 & 0,094 & 0,000 & 0,068 & 4,660 & 0,589 & 100,722 & 246 & 97 \\
\hline $25 / 1$ & 30,912 & 0,025 & 0,369 & 1,006 & 0,420 & 28,942 & 5,101 & 0,152 & 0,000 & 14,369 & 12,547 & 2,206 & 1,109 & $0,0+3$ & 0,018 & 0,000 & 0,075 & 5,114 & 0,430 & 100,999 & 12 & 97 \\
\hline $26 / 1$ & 30,828 & 0,023 & 0,259 & 1,135 & 0,881 & 27,866 & 3,153 & 0,289 & 0,000 & 14,432 & 12,377 & 2,205 & 1,415 & 0,075 & 0,055 & 0,000 & 0,084 & 4,904 & 0,506 & 100,487 & 303 & 97 \\
\hline $27 / 1$ & 32,140 & 0,000 & 0,016 & 1,123 & 3,021 & 26,599 & 2,971 & 0,764 & 0,032 & 14,024 & 11,662 & 2,017 & 1,584 & 0,160 & 0,186 & 0,021 & 0,077 & 3,851 & 0,736 & 100,982 & 292 & 102 \\
\hline $29 / 1$ & 31,419 & 0,000 & 0,008 & 1,157 & 2,047 & 27,346 & J,0SS & 0,603 & 0,032 & 14,309 & 11,817 & 2,071 & 1,486 & 0,136 & 0,121 & 0,000 & 0,104 & 4,071 & 0,745 & 100,524 & 379 & 100 \\
\hline $3 / 1$ & 29,724 & 0,077 & 0,123 & 1,101 & 2,291 & 28,601 & 3,127 & 0,615 & 0,039 & 14,220 & 11,465 & 2,066 & 1,512 & 0,131 & 0,115 & 0,009 & 0,104 & 3,919 & 0,665 & 99,902 & 404 & 108 \\
\hline $30 / 1$ & 30,752 & 0,021 & 0,000 & 1,192 & 2,732 & 27,698 & 3,033 & 0,681 & 0,030 & 14,195 & 11,623 & 2,044 & 1,583 & 0,147 & 0,161 & 0,008 & 0,086 & 4,001 & 0,827 & 100,814 & 304 & 97 \\
\hline $31 / 1$ & 29,665 & 0,116 & 0,306 & 1,139 & 1,446 & 28,100 & 3,144 & 0,377 & 0,000 & 13,195 & 12,199 & 2,292 & 1,599 & 0,101 & 0,051 & 0,000 & 0,067 & 5,155 & 0,563 & 99,513 & 227 & 92 \\
\hline $32 / 1$ & 29,063 & 0,003 & 0,112 & 1,135 & 3,368 & 27,482 & 3,054 & 0,737 & 0,041 & 13,062 & 11,670 & 2,260 & 1,830 & 0,148 & 0,163 & 0,016 & 0,074 & 4,744 & 0,580 & 99,541 & 263 & 98 \\
\hline $33 / 1$ & 31,322 & 0,000 & 0,027 & 1,343 & 3,463 & 25,787 & 2,907 & 0,823 & 0,069 & 13,439 & 11,481 & 2,102 & 1,732 & 0,194 & 0,204 & 0,036 & 0,091 & 5,007 & 0,720 & 100,745 & 293 & 87 \\
\hline $34 / 1$ & 31,229 & 0,000 & 0,002 & 1,365 & 2,184 & 27,146 & 2,952 & 0,577 & 0,023 & 14,010 & 11,734 & 2,068 & 1,507 & 0,126 & 0,111 & 0,000 & 0,143 & 4,804 & 0,910 & 100,890 & 432 & 85 \\
\hline $35 / 1$ & 30,572 & 0,000 & 0,103 & 1,174 & 2,190 & 27,258 & 3,042 & 0,566 & 0,031 & 14,081 & 11,881 & 2,122 & 1,552 & 0,125 & 0,124 & 0,000 & 0,091 & 4,976 & 0,571 & 100,457 & 315 & 94 \\
\hline $36 / 1$ & 30,909 & 0,000 & 0,008 & 1,305 & 3,289 & 26,335 & 2,933 & 0,787 & 0,046 & 13,779 & 11,474 & 2,106 & 1,672 & 0,171 & 0,187 & 0,032 & 0,078 & 4,777 & 0,751 & 100,638 & 255 & 89 \\
\hline
\end{tabular}




\begin{tabular}{|c|c|c|c|c|c|c|c|c|c|c|c|c|c|c|c|c|c|c|c|c|c|c|}
\hline $\begin{array}{c}\text { Puntos } \\
\text { de } \\
\text { análisis }\end{array}$ & $\mathbf{P}_{2} \mathbf{O}_{5}$ & $\mathrm{Al}_{2} \mathrm{O}_{3}$ & $\mathrm{SiO}_{2}$ & $\mathrm{aO}$ & $\mathbf{Y}_{2} \mathbf{O}_{3}$ & $\mathrm{e}_{2} \mathrm{O}_{3}$ & ${ }_{2} \mathrm{O}_{3}$ & $\mathrm{y}_{2} \mathrm{O}_{3}$ & $\mathrm{Ho}_{2} \mathrm{O}_{3}$ & $\mathrm{La}_{2} \mathrm{O}_{3}$ & $\mathrm{~d}_{2} \mathrm{O}_{3}$ & $\mathrm{Sm}_{2} \mathrm{O}_{3}$ & $\mathrm{Gd}_{2} \mathrm{O}_{3}$ & $\mathbf{T b}_{2} \mathbf{O}_{3}$ & $\mathrm{Er}_{2} \mathbf{O}_{3}$ & $\mathrm{Yb}_{2} \mathrm{O}_{3}$ & $\mathrm{PbO}$ & $\mathrm{ThO}_{2}$ & $\mathrm{UO}_{2}$ & Total & $\begin{array}{l}\text { Edad } \\
\text { (Ma) }\end{array}$ & Error \\
\hline \multicolumn{23}{|l|}{$\%$ peso } \\
\hline $37 / 1$ & 31,056 & 0,010 & 0,263 & 1,186 & 1,804 & 7,418 & 161 & 0,414 & 0,004 & 13,654 & 12,235 & 2,220 & 1,402 & 0,094 & 0,084 & 0,000 & 0,083 & 5,037 & 0,618 & 100,741 & 79 & 91 \\
\hline $38 / 1$ & 30,827 & 0,021 & 0,083 & 1,302 & 1,810 & 27,487 & 2,984 & 0,545 & 0,020 & 14,061 & 11,760 & 2,150 & 1,530 & 0,114 & 0,095 & 0,000 & 0,120 & 5,022 & 0,787 & 100,718 & 374 & 87 \\
\hline $39 / 1$ & 31,753 & 0,022 & 0,021 & 1,402 & 2,367 & 26,370 & 2,917 & 0,602 & 0,037 & 13,981 & 11,332 & 1,977 & 1,508 & 0,133 & 0,136 & 0,000 & 0,145 & 4,818 & 0,963 & 100,482 & 429 & 84 \\
\hline $40 / 1$ & 31,532 & 0,144 & 0,225 & 1,381 & 2,382 & 26,503 & 2,945 & 0,619 & 0,029 & 13,538 & 11,535 & 2,013 & 1,531 & 0,158 & 0,139 & 0,000 & 0,098 & 4,957 & 0,864 & 100,592 & 299 & 84 \\
\hline $41 / 1$ & 31,493 & 0,002 & 0,046 & 1,367 & 2,231 & 27,137 & 2,991 & 0,513 & 0,025 & 13,885 & 11,680 & 1,711 & 1,251 & 0,117 & 0,110 & 0,000 & 0,077 & 3,897 & 0,658 & 99,191 & 302 & 96 \\
\hline $42 / 1$ & 31,167 & 0,035 & 0,381 & 1,053 & 0,666 & 28,031 & 3,223 & 0,206 & 0,000 & 14,075 & 12,760 & 2,245 & 1,275 & 0,064 & 0,025 & 0,000 & 0,079 & 5,295 & 0,437 & 101,019 & 278 & 94 \\
\hline $43 / 1$ & 30,741 & 0,303 & 0,453 & 0,885 & 1,040 & 28,293 & 3,286 & 0,260 & 0,000 & 13,778 & 12,736 & 2,271 & 1,350 & 0,088 & 0,049 & 0,000 & 0,055 & 5,076 & 0,313 & 100,976 & 213 & 105 \\
\hline $44 / 1$ & 31,185 & 0,008 & 0,036 & 1,153 & 3,743 & 26,600 & 2,921 & 0,828 & 0,079 & 13,549 & 11,250 & 2,113 & 1,818 & 0,180 & 0,217 & 0,021 & 0,095 & 3,956 & 0,610 & 100,360 & 377 & 109 \\
\hline $45 / 1$ & 30,306 & 0,021 & 0,041 & 1,194 & 3,526 & 26,837 & 2,938 & 0,800 & 0,060 & 13,651 & 11,388 & 2,088 & 1,714 & 0,169 & 0,205 & 0,022 & 0,089 & 3,979 & 0,662 & 99,688 & 342 & 105 \\
\hline $46 / 1$ & 31,357 & 0,000 & 0,101 & 1,094 & 4,678 & 25,492 & 2,945 & 0,996 & 0,079 & 12,601 & 11,720 & 2,269 & 2,005 & 0,217 & 0,283 & 0,046 & 0,083 & 4,253 & 0,545 & 100,765 & 327 & 106 \\
\hline $48 / 1$ & 31,751 & 0,009 & 0,043 & 1,159 & 3,554 & 26,116 & 2,915 & 0,816 & 0,045 & 13,800 & 11,363 & 2,065 & 1,650 & 0,174 & 0,216 & 0,042 & 0,077 & 4,005 & 0,755 & 100,555 & 283 & 99 \\
\hline $49 / 1$ & 31,331 & 0,000 & 0,041 & 1,400 & 2,507 & 26,371 & 2,940 & 0,663 & 0,030 & 14,094 & 11,422 & 2,022 & 1,568 & 0,135 & & & 1 & 4,754 & 0,879 & 100,456 & 65 & 87 \\
\hline $5 / 1$ & 31,140 & 0,131 & 0,106 & 1,195 & 3,600 & 26,441 & 2,949 & 0,783 & 0,037 & 13,412 & 11,433 & 2,163 & 1,773 & 0,167 & 0,184 & 0,014 & 0,068 & 4,304 & 0,732 & 100,631 & 241 & 97 \\
\hline $51 / 1$ & 30,937 & 0,025 & 0,071 & 1,187 & 3,436 & 26,684 & 2,938 & 0,822 & 0,050 & 13,252 & 11,514 & 2,170 & 1,794 & 0,165 & 0,182 & 0,036 & 0,090 & 4,711 & 0,662 & 100,727 & 312 & 94 \\
\hline $52 / 1$ & 31,008 & 0,026 & 0,015 & 1,331 & 3,571 & 26,412 & 2,913 & 0,851 & 0,058 & 13,288 & 11,286 & 2,081 & 1,724 & 0,176 & 0,217 & 0,023 & 0,096 & 4,027 & 0,577 & 99,679 & 383 & 100 \\
\hline $59 / 1$ & 31,084 & 0,039 & 0,221 & 1,137 & 1,545 & 27,108 & 3,019 & 0,515 & 0,026 & 13,900 & 12,157 & 2,272 & 1,621 & 0,137 & 0,074 & 0,000 & 0,103 & 4,456 & 0,597 & 100,009 & 378 & 100 \\
\hline $6 / 1$ & 30,793 & 0,025 & 0,033 & 0,895 & 1,841 & 29,160 & 3,165 & 0,539 & 0,009 & 14,631 & 11,872 & 2,122 & 1,480 & 0,140 & 0,088 & 0,000 & 0,079 & 3,165 & 0,756 & 100,793 & 334 & 116 \\
\hline $60 / 1$ & 32,518 & 0,068 & 0,106 & 1,284 & 3,260 & 25,461 & 2,765 & 0,760 & 0,060 & 13,462 & 11,006 & 1,940 & 1,577 & 0,145 & 0,189 & 0,031 & 0,077 & 4,493 & 0,692 & 99,894 & 272 & 92 \\
\hline $62 / 1$ & 32,165 & 0,084 & 0,270 & 1,122 & 2,190 & 25,712 & 2,856 & 0,749 & 0,006 & 13,080 & 11,436 & 2,456 & 2,766 & 0,227 & 0,089 & 0,000 & 0,063 & 4,015 & 0,675 & 99,961 & 242 & 101 \\
\hline $63 / 1$ & 31,240 & 0,010 & 0,209 & 1,021 & 2,850 & 26,506 & 2,999 & 0,741 & 0,038 & 13,110 & 11,634 & 2,329 & 2,028 & 0,182 & 0,151 & 0,000 & 0,071 & 3,995 & 0,630 & 99,743 & 279 & 106 \\
\hline $7 / 1$ & 30,331 & 0,058 & 0,191 & 1,071 & 1,382 & 27,996 & 3,088 & 0,410 & 0,000 & 13,735 & 11,793 & 2,430 & 2,237 & 0,156 & 0,050 & 0,000 & 0,079 & 4,309 & 0,603 & 99,919 & 300 & 103 \\
\hline $8 / 1$ & 30,219 & 0,016 & 0,303 & 1,145 & 3,444 & 27,082 & 3,013 & 0,720 & 0,052 & 12,995 & 11,845 & 2,095 & 1,707 & 0,152 & 0,208 & 0,014 & 0,057 & 5,421 & 0,331 & 100,817 & 209 & 97 \\
\hline $9 / 1$ & 30,191 & 0,037 & 0,130 & 1,143 & 2,152 & 28,156 & 3,099 & 0,580 & 0,020 & 14,181 & 1,803 & 2,045 & 1,476 & 0,121 & 0,125 & 0,000 & 0,093 & 4,143 & 0,771 & 100,265 & 330 & 95 \\
\hline \multicolumn{23}{|c|}{ Análisis descartados: } \\
\hline $57 / 1$ & 31,247 & 0,011 & 0,028 & 1,076 & 1,977 & 27,238 & 3,074 & 0,594 & 0,027 & 14,387 & 11,850 & 2,172 & 1,585 & 0,134 & 0,103 & 0,000 & 0,161 & 4,343 & 0,879 & 100,886 & 524 & 92 \\
\hline $56 / 1$ & 29,072 & 0,000 & 1,447 & 1,390 & 2,838 & 26,474 & 2,916 & 0,708 & 0,051 & 13,345 & 11,392 & 2,064 & 1,596 & 0,169 & 0,145 & 0,013 & 0,141 & 4,855 & 0,879 & 99,495 & 431 & 86 \\
\hline $61 / 1$ & 30,493 & 2,256 & 2,925 & 1,028 & 1,778 & 24,806 & 2,761 & 0,541 & 0,011 & 12,589 & 11,019 & 2,153 & 1,893 & 0,161 & 0,072 & 0,000 & 0,063 & 4,243 & 0,624 & 99,415 & 240 & 98 \\
\hline $28 / 1$ & 26,355 & 0,000 & 3,045 & 0,821 & 1,805 & 22,915 & 2,560 & 0,392 & 0,020 & 11,750 & 10,065 & 1,602 & 1,055 & 0,100 & 0,091 & 0,000 & 0,261 & 18,039 & 0,127 & 101,003 & 334 & 36 \\
\hline
\end{tabular}


FIG. 6. Edades obtenidas a partir de las concentraciones de Th, $\mathrm{U}$ y $\mathrm{Pb}$ en monacita del gneis migmático de la muestra POT-015. El cálculo la edad media ponderada $(2 \sigma) \mathrm{se}$ obtuvo mediante el uso de Isoplot 4.15 (Ludwig, 2003).

edad antigua (524 Ma) que podría señalar herencia detrítica. La edad de 311 Ma en monacita es asignada a la del evento metamórfico de alto grado del CMC en el sector centro-sur del MNP.

\section{Discusión}

Cotejando las razones ${ }^{87} \mathrm{Sr} /{ }^{86} \mathrm{Sr}$ de los mármoles El Escorial con la curva de variación isotópica del $\mathrm{Sr}$ en el agua oceánica durante el Paleozoico-Neoproterozoico (Zaky et al., 2019 y referencias allí citadas; Fig. 7), se observa que no es posible definir de manera inequívoca una edad de sedimentación. Esto se debe a que durante el Paleozoico las razones isotópicas de Sr han experimentado más de una fluctuación. Los valores de El Escorial $\left({ }^{87} \mathrm{Sr} /{ }^{86} \mathrm{Sr}=0,70768-0,70825\right)$ podrían corresponderse a tres rangos de edades (635$625 \mathrm{Ma}$; 375-320 Ma y 300-285 Ma).

El rango de edades neoproterozoicas (635-625 Ma) puede ser descartado si se tiene en cuenta aquellas que son herencias obtenidas a partir de circones detríticos, provenientes de las unidades siliciclásticas del CMC en su localidad tipo (Hervé et al., 2016, 2018), las que registran una edad de 385 Ma para el circón clástico más joven, y acotan la edad máxima de sedimentación de esta formación al Devónico Medio ( 385-400 Ma).

El hecho que las sucesiones metasedimentarias del CMC están intruidas por granitos postmetamórficos de 286 y $261 \mathrm{Ma}$ (edades K-Ar en muscovita, Duhart et al., 2002; U-Pb en circón, Varela et al., 2005; Pankhurst et al., 2006) permiten descartar el rango de edades más jóvenes (300-285 Ma).

Estos mismos granitoides de la Formación Mamil Choique son los que intruyen a los mármoles de

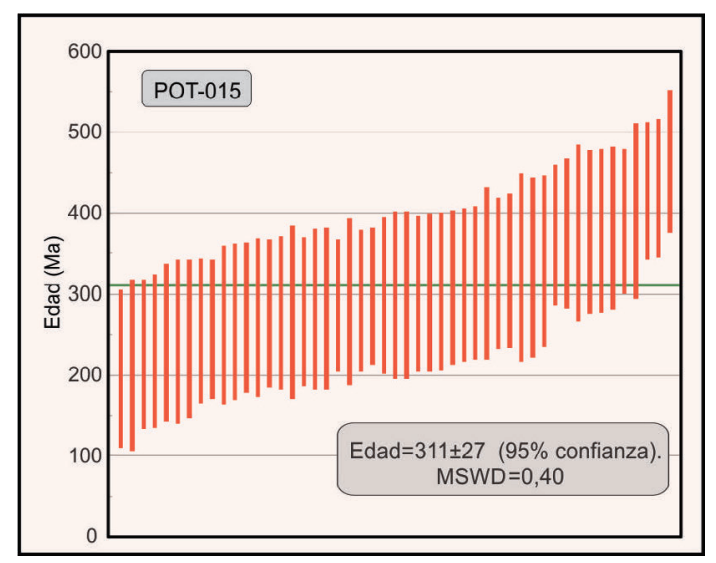

El Escorial y sucesiones metasedimentarias del $\mathrm{CMC}$, estos últimos preservados como xenolitos en afloramientos localizados inmediatamente al sur de El Escorial (Proserpio, 1987). Por otra parte, la edad de $311 \pm 27$ Ma para el evento metamórfico principal en la muestra del gneis (POT-015) acota la edad mínima de sedimentación. Además, Pankhurst et al. (2006) señalan la edad del inicio de la subducción en función del magmatismo tipo-I de edad carbonífera media (ca. $335 \mathrm{Ma}$ ). De esta manera, la posible edad de sedimentación de los carbonatos de El Escorial se ubicaría entre los 385 y 335 Ma.

Aceptando que las unidades carbonáticas estudiadas forman parte del CMC, tal como fue sugerido por Proserpio (1987), estas se habrían depositado en una plataforma mixta (carbonática-siliciclástica) de edad devónica media a carbonífera inferior. Ellas fueron involucradas con posterioridad en procesos tectónicos de un margen activo, producto del inicio de la subducción hacia el este a los ca. $335 \mathrm{Ma}$ (Pankhurst et al., 2006), y fueron metamorfizadas a los $311 \pm 27$ Ma e intruidas por el arco magmático pérmico.

Cabe la posibilidad de que los mármoles de El Escorial no tengan una relación genética con las unidades metasedimentarias del CMC, dado que no hay observaciones de campo fehacientes de que ambas litologías estén asociadas. En este caso, la edad de sedimentación podría interpretarse sobre los intervalos de aquellas más antiguas planteadas en el presente trabajo. Esto dejaría abierta la posibilidad de que los mármoles de El Escorial sean correlacionables con los estudiados por Varela et al. (2014) (Fig. 7) en la región noreste del MNP. Los mármoles de la Formación Pailemán $\left({ }^{87} \mathrm{Sr} /{ }^{86} \mathrm{Sr} \sim 0,70735 ; 625 \mathrm{Ma}\right)$ 


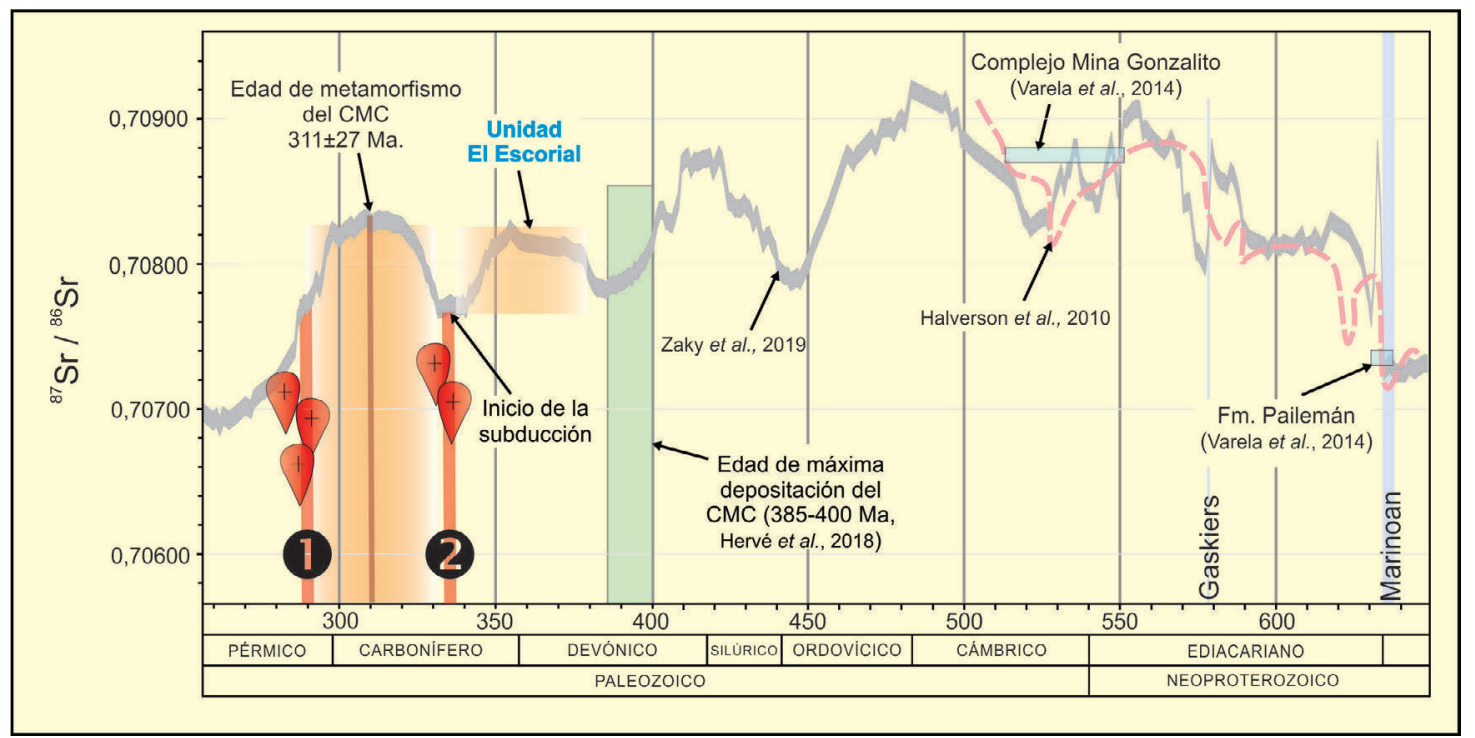

FIG. 7. Curva de variación secular de la razón isotópica de ${ }^{87} \mathrm{Sr} /{ }^{86} \mathrm{Sr}$ en el agua de mar para el Paleozoico/Ediacariano superior según recopilación de Zaky et al. (2019). Los mármoles de El Escorial indican una edad probable de sedimentación entre los 385-335 Ma, diferenciándose de otras unidades carbonáticas del MNP, con edades del Ediacariano inferior/Cámbrico inferior a medio (Varela et al., 2014). 1. Inicio del magmatismo de la Formación Mamil Choique 286-261 Ma (Duhart et al., 2002; Varela et al., 2005). 2. Inicio del magmatismo del Carbonífero medio (Pankhurst et al., 2006).

y del Complejo Mina Gonzalito $\left({ }^{87} \mathrm{Sr} /{ }^{86} \mathrm{Sr} \sim 0,70873\right.$; 550-510 Ma) fueron tentativamente referidos al Ediacariano temprano-Cámbrico inferior a medio, respectivamente (Varela et al., 2014). Esta última posibilidad implicaría extender la plataforma carbonática, generada en el Paleozoico inferior, hacia zonas más australes del MNP; no obstante, la ausencia en el sector centro-sur del MNP, de rocas ígneas y metamórficas de edades cámbricas y ordovícicas, que forman la caja de los mármoles en el sector norte del macizo (López de Luchi et al., 2010; Rapalini et al., 2013) podría sugerir que estos dos sectores poseen historias diferentes.

Por el momento, y sobre la base de la información disponible, en este trabajo sostenemos la hipótesis de que las unidades del noreste del MNP no serían correlacionables con las de la zona centro-sur (Unidad El Escorial), en concordancia con las propuestas de otros autores (Giacosa et al., 2014; Hervé et al., 2018; Renda et al., 2019, 2021; Rapela y Pankhurst, 2020; Rapela et al., 2021).

\section{Conclusiones}

Los mármoles estudiados son de tipo calcítico (calcita $>85-90 \%$ ) con contenidos menores de dolo- mita \pm flogopita \pm cuarzo \pm diópsido \pm serpentina \pm granate \pm grafito. Desde el punto de vista petrográfico, la paragénesis calcita+flogopita+diópsido+granate, registrada en los niveles impuros, indica que se alcanzaron condiciones de grado medio a alto del metamorfismo regional. Esto es consistente con el grado metamórfico que afectaron a las unidades siliciclásticas del Complejo Metamórfico Cushamen en regiones relativamente próximas a El Escorial, en las cuales la edad de metamorfismo es acotada a ca. $311 \pm 27 \mathrm{Ma}$.

Los altos tenores de $\mathrm{Ca}$ y bajos contenidos de Mn y Mg en un conjunto de muestras de mármoles indican que, en sectores específicos, estos no han sufrido importantes alteraciones postsedimentarias y que las razones isotópicas iniciales del Sr habrían permanecido lo suficientemente blindadas, validando el uso de las actuales razones isotópicas para compararlas con las variaciones seculares del agua de mar en el tiempo.

La edad de sedimentación más probable se ubica entre el Devónico Medio y el Carbonífero inferior ( 385-335 Ma).

La posibilidad de que estas rocas carbonáticas del sector central del Macizo Norpatagónico se correlacionen con las del sector nororiental son 
bajas, aunque no se descarta debido al problema de la cubierta sedimentaria que cubre los mármoles de El Escorial, y a que no ha sido posible constatar fehacientemente las relaciones de campo entre los mármoles y las metareniscas del Complejo Metamórfico Cushamen.

Los resultados obtenidos demuestran que el adecuado tratamiento y selección de muestras para la isotopía de $\mathrm{Sr}$ en metacarbonatos es una herramienta útil para la correlación entre unidades de basamento, sobre todo si se las combina con otras herramientas geocronológicas.

\section{Agradecimientos}

Los autores agradecen al LABGEO (CICTERRAUniversidad Nacional de Córdoba) y al CAI de Geocronología y Geoquímica Isotópica (Universidad Complutense de Madrid) y al grupo de investigación PAMPRE. Agradecemos también al editor, W. Vivallo, y a los revisores, S. Serra Varela y M. Márquez por las correcciones y sugerencias que mejoraron este manuscrito. El trabajo fue financiado a partir de fondos provenientes de los proyectos SECyT-UNC 18/21 No 30720150100517CB, PIP CONICET No $11220150100901 C$, FONCyT-PICT 2017-0619 y Mineco (Ministerio de Economía), España CGL2016-76439-P. A la memoria de nuestra querida amiga y compañera Carmen Galindo.

\section{Referencias}

Anselmi, G.; Gamba, M.; Panza, J. 2004. Hoja Geológica 4369-IV, Los Altares. Provincia del Chubut. Instituto de Geología y Recursos Minerales, Servicio Geológico Minero Argentino. Boletín 313: 98 p. Buenos Aires.

Brand, U.; Veizer, J. 1980. Chemical diagenesis of a multicomponent carbonate system-1: Trace elements. Journal of Sedimentary Petrology 50 (4): 1219-1236.

Cerredo, M. 1997. The metamorphism of Cushamen Formation, Río Chico area. North Patagonian Massif, Argentina. In Congreso Geológico Chileno, No. 8, Actas 2: 1236-1240. Antofagasta.

Cerredo, M.; López De Luchi, M. 1998. Mamil Choique Granitoids, southwestern North Patagonian Massif, Argentina: magmatism and metamorphism associated with a polyphasic evolution. Journal of South American Earth Sciences 11 (5): 499-515.

Dalla Salda, L.; Varela, R.; Cingolani, C. 1991. Los granitoides de Chasicó-Mencué, Macizo Norpatagónico, Rio Negro. Su implicancia geotectónica. Revista de la Asociación Geológica Argentina 46 (3-4): 189-200.
Dalla Salda, L.; Varela, R.; Cingolani, C.; Aragón, E. 1994. The Rio Chico Paleozoic crystalline complex and the evolution of North Patagonia. Journal of South American Earth Sciences 7 (3-4): 377-386.

Duhart, P.; Haller, M.; Hervé, F. 2002. Diamictitas como parte del protolito de las metamorfitas de la Formación Cushamen en Río Chico, Provincias de Río Negro y Chubut, Argentina. In Congreso Geológico Argentino, No. 15, Actas 2: 97-100. El Calafate.

Fairchild, I.; Marshall, J.; Bertrand-Sarafati, J. 1990. Stratigraphic shifts in carbon isotopes from Proterozoic stromatolitic carbonates (Mauritania): influences of primary mineralogy and diagenesis. American Journal of Science 290-A: 46-79.

Fernández González, M.; Cuesta Fernández, A.; Rubio Ordóñez, A.; Valverde-Vaquero, P. 2009. Datación química U-Th-Pb de monacita por microsonda electrónica (CHIME-EPMA Dating): Puesta a punto y resultados. In Congreso Ibérico, No.7, Congreso Nacional de Geoquímica, No. 10, Actas de trabajos: 170-177. Soria.

Fuenlabrada, J.M.; Galindo, C. 2001. Comportamiento de la relación ${ }^{87} \mathrm{Sr} /{ }^{86} \mathrm{Sr}$ en disoluciones de carbonatos impuros en función de la concentración ácida y en disoluciones de sulfatos en función del tiempo. In Congreso Ibérico de Geología, No. 3, Actas 3: 591595. Zaragoza.

Giacosa, R.; Márquez, M.; Nillni, A.; Fernández, M.; Fracchia, D.; Parisi, C.; Afonso, J.; Paredes, J.; Sciutto, J. 2004. Litología y estructura del basamento ígneometamórfico del borde SO del Macizo Nordpatagónico al oeste del río Chico, (Cushamen, Chubut, $42^{\circ} 10^{\prime} \mathrm{S}$ $\left.-70^{\circ} 30^{\prime} \mathrm{O}\right)$. Revista de la Asociación Geológica Argentina 59 (4): 569-577.

Giacosa, R.; González, P.; Silva Nieto, D.; Busteros, A.; Lagorio, S.; Rossi, A. 2014. Complejo ígneo-metamórfico Cáceres: una nueva unidad metamórfica de alto grado en el basamento de Gastre, Macizo Nordpatagónico (Chubut). In Congreso Geológico Argentino, No. 19, Actas: S21-19. Córdoba.

González, O.; Tortello, M.; Damborenea, S. 2011. Early Cambrian archaeocyathan limestone blocks in lowgrade meta-conglomerate from El Jagüelito Formation (Sierra Grande, Río Negro, Argentina). Geológica Acta 9 (2): 159-173.

González, P.; Naipauer, M.; Sato, A.; Varela, R.; Basei, M.; Cábana, M.; Vlach, S.; Arce, M.; Parada, M. 2021. Early Paleozoic structural and metamorphic evolution of the Transpatagonian Orogen related to Gondwana assembly. International Journal of Earth Sciences 110: 81-111. 
Greco, G.; González, P.; González, S.; Sato, A.; Basei, M.; Tassinari, C.; Sato, K.; Varela, R.; Llambías, E. 2015. Geology, structure and age of the Nahuel Niyeu Formation in the Aguada Cecilio area, North Patagonian Massif, Argentina. Journal of South American Earth Sciences 62: 12-32.

Greco, G.; González, S.; Sato, A.; González, P.; Basei, M.; Llambías, E.; Varela, R. 2017. The Nahuel Niyeu basin: A Cambrian forearc basin in the eastern North Patagonian Massif. Journal of South American Earth Sciences 79: 111-136

Halverson, G.; Wade, B.; Hurtgen, M.; Barovich, K. 2010. Neoproterozoic chemostratigraphy. Precambrian Research 182: 337-350.

Heredia, N.; García-Sansegundo, J.; Gallastegui, G.; Farias, P.; Giacosa, R.; Hongn, F.; Tubía, J.; Alonso, J.; Busquets, P.; Charrier, R.; Clariana, P.; Colombo, F.; Cuesta, A.; Gallastegui, J.; Giambiagi, L.; GonzálezMenéndez, L.; Limarino, C.; Martín González, F.; Pedreira, D.; Quintana, L.; Rodríguez-Fernández, L.; Rubio-Ordóñez, A.; Seggiaro, R.; Serra Varela, S.; Spalletti, L.; Cardó, R.; Ramos, V. 2018. The PreAndean phases of construction of the Southern Andes basement in Neoproterozoic-Paleozoic times. In The Evolution of the Chilean-Argentinean Andes (Folguera, A.; Contreras Reyes, E.; Heredia, N.; Encinas, A.; Iannelli, S.B.; Oliveros, V.; Dávila, F.M.; Collo, G.; Giambiagi, L.; Maksymowicz, A.; Iglesia Llanos, M.P.; Turienzo, M.; Naipauer, M.; Orts, D.; Litvak, V.D.; Álvarez, O.; Arriagada, C.; editors). Springer: 111-131.

Hervé, F.; Haller, M.; Duhart, P.; Fanning, M. 2005. SHRIMP U-Pb ages of detrital zircons from Cushamen and Esquel formations, North Patagonian Massif, Argentina: geological implications. In Congreso Geológico Argentino, No. 16, Actas: 309-314. La Plata.

Hervé, F.; Calderón, M.; Fanning, C.M.; Pankhurst, R.J.; Fuentes, F.; Rapela, C.W.; Correa, J.; Quezada, P.; Marambio, C. 2016. Devonian magmatism in the accretionary complex of southern Chile. Journal of the Geological Society London 173 (4): 587-602.

Hervé, F.; Calderón, M.; Fanning, C.M.; Pankhurst, R.J.; Rapela, C.W.; Quezada, P. 2018. The country rocks of Devonian magmatism in the North Patagonian Massif and Chaitenia. Andean Geology 45 (3): 301-317. doi: 10.5027/andgeoV45n3-3117.

Jacobsen, S.; Kaufman, A. 1999. The Sr, C and O isotopic evolution of Neoproterozoic seawater. Chemical Geology 161: 37-57.

Jiang, G.; Kaufman, A.; Christie-Blick, N.; Zhang, S.; Wu, H. 2007. Carbon isotope variability across the Ediacaran
Yangtze platform in South China: Implications for a large surface-to-deep ocean $\delta^{13} \mathrm{C}$ gradient. Earth and Planetary Science Letters 261: 303-320.

Lesta, P.; Mainardi, E.; Stubelj, R. 1980. Plataforma continental argentina. In Simposio de Geología Regional Argentina, No. 2, Academia Nacional de Ciencias Córdoba 2: 1577-1602. Córdoba.

López de Luchi, M.; Rapalini, A.; Tomezzoli, R. 2010. Magnetic Fabric and microstructures of Late Paleozoic granitoids from the North Patagonian Massif: Evidence of a collision between Patagonia and Gondwana? Tectonophysics 494: 118-137.

López de Luchi, M.; Rapalini, A.; Martínez Dopico, C.; Cerredo, M.; Wemmer, K. 2011. Insights into the Paleozoic tectonics evolution of the North Patagonian Massif: Start up -hypothesis on a sequence of the collisional events. In Congreso Geológico Argentino, No. 18, S1-La Patagonia en el contexto geodinámico de Gondwana: 103-104. Neuquén.

Lucassen, F.; Trumbull, R.; Franz, G.; Creixell, C.; Vásquez, P.; Romer, R.; Figueroa, O. 2004. Distinguishing crustal recycling and juvenile additions at active continental margins: the Paleozoic to recent compositional evolution of the Chilean Pacific margin (36-41 $\left.{ }^{\circ} \mathrm{S}\right)$. Journal of South American Earth Sciences 17 (2): 103-119.

Ludwig, K. 2003. Isoplot/ExVersion 4.15, a Geochronological Toolkit for Microsoft Excel. Berkeley Geochronology Center, Special Publication 4.

Martínez Dopico, C.; López de Luchi, M.; Rapalini, A.; Kleinhanns, I. 2011. Crustal segments in the North Patagonian Massif, Patagonia: an integrated perspective based on Sm-Nd isotope systematics. Journal of South American Earth Science 31: 324-341.

Melezhik, V.A.; Gorokhov, I.M.; Fallick, A.E.; Gjelle, S. 2001. Strontium and carbon isotope geochemistry applied to dating of carbonate sedimentation: an example from high-grade rocks of the Norwegian Caledonides. Precambrian Research 108 (3-4): 267-292.

Montañez, I.; Osleger, D.; Banner, J.; Mack, L.; Musgrove, M. 2000. Evolution of the $\mathrm{Sr}$ and $\mathrm{C}$ isotope composition of Cambrian oceans. Geological Society of America Today 10 (5): 1-5.

Montel, J.M.; Foret, S.; Veschambre, M.; Nicollet, C.; Provost, A. 1996. Electron microprobe dating of monazite. Chemical Geology 131 (1-4): 37-53.

Murra, J.; Baldo, E.; Verdecchia, S.; Galindo, C. 2017. Los mármoles cálcicos de El Escorial (Formación Cushamen), macizo Nord-Patagónico: caracterización isotópica de ${ }^{87} \mathrm{Sr}-{ }^{86} \mathrm{Sr}$ y probable edad de sedimentación. In Congreso Geológico Argentino, No. 20, S15 La geo- 
cronología como herramienta para la resolución de problemas geológicos y tectónicos: 82-84. Tucumán.

Ostera, H.A.; Linares, E.; Haller, M.J.; Cagnoni, M.C.; López de Luchi, M. 2001. A widespread metamorphic Devonian episode in Northern Patagonia, Argentina. In South American Symposium on Isotope Geology, No. 3, Actas CD: 52, Resúmenes: 161. Santiago.

Pankhurst, R.; Rapela, C.; Fanning, C.; Márquez, M. 2006. Gondwanide continental collision and the origin of Patagonia. Earth-Science Reviews 76: 235-257.

Pankhurst, R.J.; Rapela, C.W.; López De Luchi, M.G.; Rapalini, A.E.; Fanning, C.M.; Galindo, C. 2014. The Gondwana connections of northern Patagonia. Journal of the Geological Society 171: 313-328.

Prokoph, A.; Shields, G.; Veizer, J. 2008. Compilation and time-series analysis of a marine carbonate $\delta^{18} \mathrm{O}$, $\delta^{13} \mathrm{C},{ }^{87} \mathrm{Sr} /{ }^{86} \mathrm{Sr}$ and $\delta^{34} \mathrm{~S}$ database through Earth history. Earth-Science Reviews 87: 113-133.

Proserpio, C. 1987. Descripción Geológica de la Hoja 44e, Valle General Racedo (1:200.000), provincia de Chubut. Dirección Nacional de Minería y Geología, Boletín 201: 1-102. Argentina.

Ramos, V. 2008. Patagonia: a paleozoic continent adrift? Journal of South American Earth Science 26: 235-251.

Ramos, V.; Naipauer, M. 2014. Patagonia: where does it come from? Journal of Iberian Geology 40 (2): 367-379.

Rapalini, A.; López de Luchi, M.; Martínez Dopico, C.; Lince Klinger, F.; Giménez, M., Martínez, P. 2010. Did Patagonia collide against Gondwana in the Late Paleozoic? Some insights from a multidisciplinary study of magmatic units of the North Patagonian Massif. Geológica Acta 8: 349-371.

Rapalini, A.; López de Luchi, M.; Tohver, E.; Cawood, P. 2013. The South American ancestry of the North Patagonian Massif: geochronological evidence for an autochthonous origin? Terra nova 25: 337-342.

Rapela, C.W.; Pankhurst, R.J. 2020. The continental crust of northeastern Patagonia. Ameghiniana 57 (5): 480-498.

Rapela, C.; Hervé, R.; Pankhurst, R.; Calderón, M.; Fanning, C.; Quezada, P.; Poblete, F.; Palape, C.; Reyes, T. 2021. The Devonian accretionary orogen of the North Patagonian cordillera. Gondwana Research 96: 1-21.

Rasband, W. 2017. ImageJ, U.S. National Institutes of Health, Bethesda, Maryland, USA, https://imagej. nih.gov/ij/ (Última visita 12/01/2022).

Renda, E.; Álvarez, D.; Prezzi, C.; Oriolo, S.; Vizán, H. 2019. Inherited basement structures and their influence in foreland evolution: A case study in Central Patagonia, Argentina. Tectonophysics: $772 \mathrm{p}$.
Renda, E.; González, P.; Vizán, H.; Oriolo, S.; Prezzi, C.; Ruiz González, V.; Schulz, B.; Krause, J.; Basei, M. 2021. Igneous-metamorphic basement of Taquetrén Range, patagonia, Argentina: A key locality for the reconstruction of the paleozoic evolution of Patagonia. Journal of South American Earth Sciences 106: 103045.

Serra-Varela, S.; Heredia, N.; Giacosa, R.; García-Sansegundo, J.; Farías, P. 2020. Review of the polyorogenic Palaeozoic basement of the Argentinean North Patagonian Andes: age, correlations, tectonostratigraphic interpretation and geodynamic evolution. International Geology Review, doi: 10.1080/00206814.2020.1839798.

Scherrer, N.C.; Engi, M.; Gnos, E.; Jakob, V.; Leichti, A. 2000. Monazite analysis; from sample preparation to microprobe age dating and REE quantification. Schweizerische Mineralogische und Petrographische Mitteilungen 80: 93-105.

Varela, R.; Basei, M.; Cingolani, C.; Siga Jr., O.; Passarelli, C. 2005. El Basamento Cristalino de los Andes norpatagónicos en Argentina: geocronología e interpretación tectónica. Revista Geológica de Chile 32: 167-182. doi: 10.5027/andgeoV32n2-a01.

Varela, R.; González, P.; Philipp, R.; Sato, A.; González, S.; Greco, G.; Naipauer, M. 2014. Isótopos de estroncio en calcáreos del noreste Patagónico: resultados preliminares. Revista de la Asociación Geológica Argentina 71 (4): 526-536.

Veizer, J.; Ala, D.; Azmy, K.; Bruckschen, P.; Buhl, D.; Bruhn, F.; Carden, G.A.F.; Diener, A.; Ebneth, S.; Goddéris, Y.; Jasper, T.; Korte, C.; Pawellek, F.; Podlaha, O.G.; Strauss, H. 1999. ${ }^{87} \mathrm{Sr} /{ }^{86} \mathrm{Sr},{ }^{13} \mathrm{C}$ and ${ }^{18} \mathrm{O}$ evolution of Phanerozoic seawater. Chemical Geology 161: 59-88.

Volkheimer, W. 1964. Estratigrafía de la zona extraandina del Departamento de Cushamen (Chubut) entre los paralelos $42^{\circ}$ y $42^{\circ} 30^{\prime}$ y los meridianos $70^{\circ}$ y $72^{\circ}$. Revista de la Asociación Geológica Argentina 19 (2): 85-107.

Whitney, D.L.; Evans, B.W. 2010. Abbreviations for names of rock-forming minerals. American Mineralogist 95: 185-187.

Williams, M.L.; Jercinovic, M.J.; Terry, M.P. 1999. Age mapping and dating of monazite on the electron microprobe: Deconvoluting multistage tectonic histories. Geology 27 (11): 1023-1026.

Zaky, A.; Brand, U.; Buhl, D.; Blamey, N.; Bitner, M.; Logan, A.; Gaspard, D.; Popov, A. 2019. Strontium isotope geochemistry of modern and ancient archives: tracer of secular change in ocean chemistry. Canadian Journal of Earth Sciences 56 (3): 245-264. 